\title{
Extended Higgs sector of 2HDM with real singlet facing LHC data
}

\author{
A. Arhrib ${ }^{1}$, R. Benbrik ${ }^{2}$, M. EL Kacimi ${ }^{3}$, L. Rahili ${ }^{4}$, S. Semlali ${ }^{3}$, \\ ${ }^{1}$ Faculty of Sciences and Techniques, Abdelmalek Essaadi University, B.P. 416, Tanger, Morocco \\ ${ }^{2}$ MSISM Team, Faculté Polydisciplinaire de Safi, Sidi Bouzid, B.P. 4162, Safi, Morocco \\ ${ }^{3}$ LPHEA, Faculty of Science Semlalia, Cadi Ayyad University, P.O.B. 2390, Marrakech, Morocco \\ ${ }^{4}$ EPTHE, Faculty of Sciences, Ibn Zohr University, P.O.B. 8106, Agadir, Morocco
}

Received: 3 December 2018 / Accepted: 8 November 2019 / Published online: 8 January 2020

(C) The Author(s) 2020

\begin{abstract}
We study the phenomenology of the two Higgs doublet model with a real singlet scalar $S$ (N2HDM). The model predicts three CP-even Higgses $h_{1,2,3}$, one CP-odd $A^{0}$ and a pair of charged Higgs. We discuss the consistency of the N2HDM with theoretical as well as with all available experimental data. In contrast with previous studies, we focus on the scenario where $h_{2}$ is the Standard Model (SM) 125 $\mathrm{GeV}$ Higgs, while $h_{1}$ is lighter than $h_{2}$ which may open a window for Higgs to Higgs decays. We perform an extensive scan into the parameter space of N2HDM of type I and explore the effect of the singlet-doublet admixture. We found that a large singlet-doublet admixture is still compatible with the recent Higgs data from LHC. Moreover, we show that $h_{1}$ could be quasi-fermiophobic and would decay dominantly into two photons. We also study in details the consistency of the non-detected decay of $h_{2} \rightarrow h_{1} h_{1}$ with LHC data followed by $h_{1} \rightarrow \gamma \gamma$ which leads to four photons final state at LHC: $p p \rightarrow h_{2} \rightarrow h_{1} h_{1} \rightarrow 4 \gamma$. Using the results of null searches of multi-photons carried by the ATLAS collaboration, we have found that a large area of the parameter space is still allowed. We also demonstrate that various neutral Higgs of the N2HDM could have several exotic decays.
\end{abstract}

\section{Introduction}

A Higgs boson has been discovered in the first run of Large Hadron Collider (LHC) with 7 and $8 \mathrm{TeV}$ energy in 2012 $[1,2]$ and some of its properties have been established. During the second run of LHC with $13 \mathrm{TeV}$ center of mass energy, some measurements of the properties of the Higgs boson have improved [3-7] and new observations such as $h \rightarrow b \bar{b}$ and $p p \rightarrow t \bar{t} h$ have been reported [8-10].

The aforementioned Higgs boson properties established so far will be further improved by the High Luminosity pro-

a e-mail: s.seemlali@gmail.com gram of the future LHC run (HL-LHC) [11-14]. At the HLLHC, one can pin down the uncertainties on the Higgs boson couplings to a few percent level in some cases [15] and provide indirect hints to the awaited new physics. Moreover, in the clean environment of the $e^{+} e^{-}$collider such as ILC and CEPC, which can act like a Higgs factory, one can improve the Higgs boson measurements [18,19].

Although all data collected by LHC seem to indicate that the Higgs boson particle is in perfect agreement with the SM predictions, there are many theoretical as well as experimental indications which point out that the SM is only an effective theory at low scale of a more fundamental one. One common feature of those Beyond SM (BSM) theories is an extended Higgs sector with an extra singlet, doublet, and/or triplet. Most of the higher Higgs representations with an extra doublet/singlet predict in their spectrum extra neutral and/or charged Higgs states. A discovery of another or several additional Higgs bosons would be considered as a clear evidence of an extended Higgs sector and a departure from the SM.

Following the discovery of a Higgs boson, there has been a large number of works dedicated to extending the SM Higgs sector by extra Higgs fields. Among the simplest one we mention:

A real singlet scalar that has a mixing with the SM Higgs boson [20], the popular two Higgs Doublet Model (2HDM) [21-23] with or without natural flavor conservation and the inert Higgs model that provides dark matter candidate [2427]. Recently, there have been also phenomenological studies that extend the $2 \mathrm{HDM}$ with an additional real gauge-singlet scalar which acts like dark matter candidate [28,29]. One can also extend the 2HDM by adding a real scalar singlet with non-vanishing expectation value that can mix with the doublets $[30,31]$. These studies referred to this model as the next-to-minimal 2HDM (N2HDM).

In the two variants of the N2HDM, the scalar spectrum is richer than the traditional 2HDM, which implies an interest- 
ing phenomenology at colliders including but not restricted to scalar-to-scalar decays, exotic decays and fermiophobic scenarios which are precluded in the SM and occur hardly in the 2HDM. The model can easily accommodate a SM-like Higgs Boson that easily satisfies all the constraints from LHC measurements. Despite the existence of mixing among CP-even mass eigenstates, which would modify the SM-like Higgs couplings to fermions and gauge bosons, constraints from signal strength measurements can be easily satisfied (within the present range of systematic and statistical errors).

In the N2HDM, the scalar spectrum contains $3 \mathrm{CP}$-even states $h_{1,2,3}$, one CP-odd $A$ and a pair of charged Higgs $H^{ \pm}$. $h_{1,2,3}$ are admixtures of the doublets and the singlet components while $A$ and $H^{ \pm}$are purely made of the doublets components.

A comprehensive analysis of the N2HDM has been carried by the authors of Ref. [31]. A general scan was performed over the parameter space to look for possible scenarios that allow any of the neutral Higgs bosons $h_{i}$ to be identified with the discovered Higgs boson particle at the LHC. It is found that a large singlet-doublet admixture is still compatible with LEP and all LHC data, while satisfying all theoretical and experimental constraints [31]. In the present study, we would like to do a comprehensive study of N2HDM type-I for the case where $h_{2}$ is the $125 \mathrm{GeV}$ Higgs while $h_{1}$ and $A$ are lighter than $h_{2}$. This scenario opens a new window for beyond Standard Model decays of $h_{2}$ such as $h_{2} \rightarrow h_{1} h_{1}$ and probably $h_{2} \rightarrow A A, A A^{*}, Z^{*} A, W^{*} H^{ \pm}$which are still compatible with Higgs data.

Furthermore, we will study the possibility where the light Higgs $h_{1}$ can be partially fermiophobic in N2HDM typeI as well as the consistency of the non-detected decay of $h_{2} \rightarrow h_{1} h_{1}$ with LHC data followed by $h_{1} \rightarrow \gamma \gamma$ which leads to four photons final state at LHC [32].

We will also present some decays of the heavy Higgs $\left(h_{3}\right)$ into Standard Model particles and other decays to scalar-scalar and scalar-vector boson $\left(h_{3} \rightarrow h_{1} h_{1}, h_{3} \rightarrow\right.$ $\left.h_{1} h_{2}, h_{3} \rightarrow A Z, h_{3} \rightarrow H^{ \pm} W^{\mp}\right)$

The paper is organized as follow: Sect. 2 is devoted to the N2HDM and its parametrization, in Sect. 3 we review the theoretical and experimental constraints that the N2HDM is subject to. We present our numerical result in Sect. 4 and conclude in Sect. 5. Several technical details are given in the Appendix.

\section{The 2HDM with a real singlet field: N2HDM}

In this section, we present a review of the N2HDM. We discuss the scalar potential and derive the spectrum and the parametrization of the model. We also present the Yukawa textures and discuss the natural flavor conservation of the model. Couplings of the Higgs bosons to gauge bosons are also shown and their sum rules are discussed.

\subsection{The Higgs potential}

The scalar sector of N2HDM consists of two weak isospin doublets $H_{i}(\mathrm{i}=1,2)$, with hypercharge $Y=1$ and a real singlet field with hypercharge $Y=0$ which are given by

$H_{i}=\left(\begin{array}{c}\phi_{i}^{ \pm} \\ \frac{1}{\sqrt{2}}\left(v_{i}+\phi_{i}+i \chi_{i}\right)\end{array}\right)$ and $S=\frac{1}{\sqrt{2}}\left(v_{s}+\phi_{s}\right)$

The most general renormalizable scalar potential for the model that respect $S U(2)_{L} \otimes U(1)_{Y}$ gauge symmetry has the following form:

$$
\begin{aligned}
V\left(H_{1}, H_{2}, S\right)= & m_{11}^{2} H_{1}^{\dagger} H_{1}+m_{22}^{2} H_{2}^{\dagger} H_{2} \\
& -\mu^{2}\left(H_{1}^{\dagger} H_{2}+H_{2}^{\dagger} H_{1}\right)+\frac{1}{2} m_{S}^{2} S^{2} \\
& +\frac{\lambda_{1}}{2}\left(H_{1}^{\dagger} H_{1}\right)^{2}+\frac{\lambda_{2}}{2}\left(H_{2}^{\dagger} H_{2}\right)^{2} \\
& +\lambda_{3} H_{1}^{\dagger} H_{1} H_{2}^{\dagger} H_{2}+\lambda_{4} H_{1}^{\dagger} H_{2} H_{2}^{\dagger} H_{1} \\
& +\frac{\lambda_{5}}{2}\left[\left(H_{1}^{\dagger} H_{2}\right)^{2}+\left(H_{2}^{\dagger} H_{1}\right)^{2}\right] \\
& +\frac{\lambda_{6}}{8} S^{4}+\frac{1}{2}\left[\lambda_{7} H_{1}^{\dagger} H_{1}+\lambda_{8} H_{2}^{\dagger} H_{2}\right] S^{2}
\end{aligned}
$$

where $m_{11}^{2}, m_{22}^{2}$ and $m_{S}^{2}$ are the mass parameters. By hermiticity of the scalar potential $\lambda_{1,2,3,4,6,7,8}$ are dimensionless real parameters while $\lambda_{5}$ and $\mu^{2}$ can be complex to allow CP violation in the scalar sector. In the present study, we assume that all scalar parameters are real. Therefore, the only source of CP violation is in the Cabbibo-KobayashiMaskawa matrix. We remind here that we allow a dimension 2 term $\mu^{2}$ which break softly $Z_{2}$ symmetry. This discrete $Z_{2}$ symmetry is usually imposed in order to avoid Flavor Changing Neutral Current (FCNC) at tree level in the Yukawa sector.

Assuming that spontaneous electroweak symmetry breaking (EWSB) is taking place at some electrically neutral point in the field space, and denoting the corresponding VEVs by

$$
\left\langle H_{1}\right\rangle=\frac{1}{\sqrt{2}}\left(\begin{array}{c}
0 \\
v_{1}
\end{array}\right),\left\langle H_{2}\right\rangle=\frac{1}{\sqrt{2}}\left(\begin{array}{c}
0 \\
v_{2}
\end{array}\right) \text { and }\langle S\rangle=\frac{1}{\sqrt{2}} v_{S}
$$

The parameters $m_{11}^{2}, m_{22}^{2}$ and $m_{S}^{2}$ can be eliminated by the minimization conditions of the potential Eq. (2):

$$
\begin{aligned}
& m_{11}^{2}=\mu^{2} t_{\beta}-\frac{1}{2} \lambda_{1} v^{2} c_{\beta}^{2}-\frac{1}{2} \lambda_{345} v^{2} s_{\beta}^{2}-\frac{1}{4} \lambda_{7} v_{S}^{2} \\
& m_{22}^{2}=\mu^{2} t_{\beta}^{-1}-\frac{1}{2} \lambda_{1} v^{2} s_{\beta}^{2}-\frac{1}{2} \lambda_{345} v^{2} c_{\beta}^{2}-\frac{1}{4} \lambda_{8} v_{S}^{2} \\
& m_{S}^{2}=-\frac{1}{2} \lambda_{7} v^{2} c_{\beta}^{2}-\frac{1}{2} \lambda_{8} v^{2} s_{\beta}^{2}-\frac{1}{4} \lambda_{6} v_{S}^{2}
\end{aligned}
$$


where $s_{x}, c_{x}, t_{x}$ stand for $\sin x, \cos x$ and $\tan x$ respectively, and $\lambda_{345}=\lambda_{3}+\lambda_{4}+\lambda_{5}$ and $t_{\beta}=v_{2} / v_{1}$.

After the EWSB of $S U(2)_{L} \otimes U(1)_{Y}$ down to electromagnetic $U(1)$, three of the nine Higgs degrees of freedom corresponding to the Goldstone bosons are absorbed by the longitudinal components of vector boson $W^{ \pm}$and $Z^{0}$. The remaining six degrees of freedom should manifest as physical Higgses: three CP-even scalars $\left(h_{1}, h_{2}, h_{3}\right.$ with $m_{h_{1}}<m_{h_{2}}<m_{h_{3}}$ ), one CP-odd $A$ and a charged Higgs pair $H^{ \pm}$.

\subsection{Higgs masses and mixing angles}

The most general form of the squared mass matrix $7 \times 7$ of the Higgs sector can be recast, using Eqs. (4-6), into a block diagonal form of three submatrices: one $3 \times 3$ matrix denoted in the following by $\mathcal{M}_{\mathcal{C} \mathcal{P}_{\text {even }}}^{2}$ for $\mathrm{CP}$-even sector, one $2 \times 2$ matrix $\mathcal{M}_{\mathcal{C} \mathcal{P}_{\text {odd }}}^{2}$ for $\mathrm{CP}$-odd sector and one $2 \times 2$ matrix denoted by $\mathcal{M}_{ \pm}^{2}$ for the charged sector.

The squared mass matrix for the charged fields $\phi_{1,2}^{ \pm}$is:

$\mathcal{M}_{ \pm}^{2}=\left(\begin{array}{ll}\mu^{2} t_{\beta}-\frac{1}{2} \lambda_{45}^{+} v^{2} s_{\beta}^{2} & -\mu^{2}+\frac{1}{2} \lambda_{45}^{+} v^{2} s_{\beta} c_{\beta} \\ -\mu^{2}+\frac{1}{2} \lambda_{45}^{+} v^{2} s_{\beta} c_{\beta} & \mu^{2} t_{\beta}^{-1}-\frac{1}{2} \lambda_{45}^{+} v^{2} c_{\beta}^{2}\end{array}\right)$

with $\lambda_{45}^{+}=\lambda_{4}+\lambda_{5}$. This matrix is diagonalized by the following orthogonal matrix $\mathcal{R}_{\beta}$, given by :

$\mathcal{R}_{\beta}=\left(\begin{array}{cc}c_{\beta} & -s_{\beta} \\ s_{\beta} & c_{\beta}\end{array}\right)$

Among the two eigenvalues of $\mathcal{M}_{ \pm}^{2}$, one is zero and corresponds to the charged Goldstone boson $G^{ \pm}$while the other one corresponds to the charged Higgs boson $H^{ \pm}$and is given by:

$m_{H^{ \pm}}^{2}=\frac{1}{s_{2 \beta}}\left[2 \mu^{2}-\frac{1}{2} \lambda_{45}^{+} v^{2} s_{2 \beta}\right]$

The charged Higgs $H^{ \pm}$and the charged goldstone $G^{ \pm}$are orthogonal rotation of the weak eigenstates $\phi_{1}^{ \pm}, \phi_{2}^{ \pm}$,

$G^{ \pm}=c_{\beta} \phi_{1}^{ \pm}+s_{\beta} \phi_{2}^{ \pm}, \quad H^{ \pm}=-s_{\beta} \phi_{1}^{ \pm}+c_{\beta} \phi_{2}^{ \pm}$

The neutral scalar and pseudo-scalar mass matrices are given by:

$\mathcal{M}_{\mathcal{C} \mathcal{P}_{\text {even }}}^{2}=\left(\begin{array}{lll}\mu^{2} t_{\beta}+\lambda_{1} v^{2} c_{\beta}^{2} & -\mu^{2}+\lambda_{345} v^{2} s_{\beta} c_{\beta} & \frac{\lambda_{7} v v_{S} c_{\beta}}{2 \sqrt{2}} \\ -\mu^{2}+\lambda_{345} v^{2} s_{\beta} c_{\beta} & \mu^{2} t_{\beta}^{-1}+\lambda_{2} v^{2} s_{\beta}^{2} & \frac{\lambda_{8} v v_{S} s_{\beta}}{2 \sqrt{2}} \\ \frac{\lambda_{7} v v_{S} c_{\beta}}{2 \sqrt{2}} & \frac{\lambda_{8} v v_{S} s_{\beta}}{2 \sqrt{2}} & \frac{\lambda_{6} v_{S}^{2}}{8}\end{array}\right)$

and

$\mathcal{M}_{\mathcal{C P}_{\text {odd }}}^{2}=\left(\begin{array}{ll}\mu^{2} t_{\beta}-\lambda_{5} v^{2} s_{\beta}^{2} & -\mu^{2}+\lambda_{5} v^{2} s_{\beta} c_{\beta} \\ -\mu^{2}+\lambda_{5} v^{2} s_{\beta} c_{\beta} & \mu^{2} t_{\beta}^{-1}-\lambda_{5} v^{2} c_{\beta}^{2}\end{array}\right)$
The physical states $h_{i}=\left\{h_{1}, h_{2}, h_{3}\right\}$ are obtained by an orthogonal transformation $h_{i}=\mathcal{R}_{\alpha_{1,2,3}} \phi_{i},(i=1,2, s)$ that diagonalizes the mass matrix $\mathcal{M}_{\mathcal{C} \mathcal{P}_{\text {even }}}^{2}$,

$$
\left(\begin{array}{l}
h_{1} \\
h_{2} \\
h_{3}
\end{array}\right)=\mathcal{R}_{\alpha_{1,2,3}}\left(\begin{array}{l}
\phi_{1} \\
\phi_{2} \\
\phi_{s}
\end{array}\right)
$$

with:

$\mathcal{R}_{\alpha_{1,2,3}}=\left(\begin{array}{lll}c_{1} c_{2} & s_{1} c_{2} & s_{2} \\ -c_{1} s_{2} s_{3}-s_{1} c_{3} & c_{1} c_{3}-s_{1} s_{2} s_{3} & c_{2} s_{3} \\ -c_{1} s_{2} c_{3}+s_{1} s_{3}-c_{1} s_{3}-s_{1} s_{2} c_{3} & c_{2} c_{3}\end{array}\right)$

with $s_{i}=\sin \alpha_{i} \quad c_{i}=\cos \alpha_{i}$.

Without loss of generality we assume that $m_{h_{1}}<m_{h_{2}}<$ $m_{h_{3}}$.

From Eq. (12), it is easy to get the two eigenvalues of $\mathcal{M}_{\mathcal{C} \mathcal{P}_{\text {odd }}}^{2}$, one is vanishing and corresponds to the neutral Goldstone boson $G^{0}$ while the other one corresponds to the pseudo-scalar $A$ :

$m_{A}^{2}=\frac{1}{s_{\beta} c_{\beta}}\left[\mu^{2}-\lambda_{5} v^{2} s_{\beta} c_{\beta}\right]$

The CP-odd state $A$ and the neutral Goldstone $G^{0}$ are obtained by an orthogonal rotation of the weak eigenstates $\chi_{1}, \chi_{2}$ :

$G^{0}=c_{\beta} \chi_{1}+s_{\beta} \chi_{2}, \quad A=-s_{\beta} \chi_{1}+c_{\beta} \chi_{2}$.

\subsection{Yukawa texture}

There are different types of Higgs couplings to fermions. The general structure of the Yukawa Lagrangian when both Higgs fields couple to all fermions is given by:

$$
\begin{aligned}
\mathcal{L}_{Y}= & \bar{Q}_{L}^{0} \tilde{\Phi}_{2} \eta_{2}^{U, 0} U_{R}^{0}+\bar{Q}_{L}^{0} \Phi_{2} \eta_{2}^{D, 0} D_{R}^{0}+\bar{Q}_{L}^{0} \tilde{\Phi}_{1} \eta_{1}^{U, 0} U_{R}^{0} \\
& +\bar{Q}_{L}^{0} \Phi_{1} \eta_{1}^{D, 0} D_{R}^{0}+\text { h.c }
\end{aligned}
$$

where $Q_{L}^{0}$ is the weak isospin quark doublet, $U_{R}^{0}$ and $D_{R}^{0}$ are the weak isospin quark singlets and $\eta_{1,2}^{U, 0}, \eta_{1,2}^{D, 0}$ are matrices in flavor space, then the above Lagrangian will generate Flavor Changing Neutral Currents (FCNC) at tree level which can invalidate some low energy observables in $\mathrm{B}, \mathrm{D}$ and $\mathrm{K}$ physics. In order to avoid such FCNC, it is customary to invoke a $Z_{2}$ symmetry that forbids FCNC couplings at tree level [33]. Depending on the $Z_{2}$ assignment, we have four types of Yukawa interactions [34]. In the present study, we focus only on type-I where all fermions couple only to one of the two Higgs doublets. In this case:

$$
\begin{aligned}
\mathcal{L}_{Y}^{I}= & \bar{Q}_{L}^{0} \tilde{\Phi}_{2} \eta_{2}^{U, 0} U_{R}^{0}+\bar{Q}_{L}^{0} \Phi_{2} \eta_{2}^{D, 0} D_{R}^{0} \\
& +\bar{U}_{R}^{0} \eta_{2}^{U, 0 \dagger} \tilde{\Phi}_{2}^{\dagger} Q_{L}^{0}+\bar{D}_{R}^{0} \eta_{2}^{D, 0 \dagger} \Phi_{2}^{+} Q_{L}^{0}
\end{aligned}
$$


Neutral Higgs couplings to a pair of fermions are:

$g_{h_{1} f \bar{f}}: \frac{\mathcal{R}_{12}}{s_{\beta}}=\frac{c_{2} s_{1}}{s_{\beta}}$

$g_{h_{2} f \bar{f}}: \frac{\mathcal{R}_{22}}{s_{\beta}}=\frac{\left(c_{1} c_{3}-s_{1} s_{2} s_{3}\right)}{s_{\beta}}$

$g_{h_{3} f \bar{f}}: \frac{\mathcal{R}_{32}}{s_{\beta}}=-\frac{\left(c_{1} s_{3}+s_{1} s_{2} c_{3}\right)}{s_{\beta}}$

where $f$ designate any type of fermions.

\subsection{Higgs couplings to gauge bosons and sum rules}

We present shortly here the Higgs couplings to gauge bosons and discuss the sum rules required by unitarity $[35,36]$ which could be derived either from the Lagrangian or using unitarity arguments by considering the processes $f f \rightarrow V V$ and requiring that the term that increases with energy should cancel (see Ref. [37]). In Refs. [36] and [37], the sum rules are established for multi-Higgs doublet models using unitarity arguments for scattering amplitudes and also unitarity for the mixing matrix. In our case of N2HDM, as we will see below, these sum rules will apply as a consequence of the unitarity of the orthogonal matrix $R_{i j}$. Just before presenting these sum rules, we also refer to the normalized couplings of neutral Higgs to a pair of gauge bosons $V=Z, W$ that are given by:

$g_{h_{1} V V}: \quad c_{\beta} \mathcal{R}_{11}+s_{\beta} \mathcal{R}_{12}=c_{\alpha_{2}} c_{\beta-\alpha_{1}}$,

$g_{h_{2} V V}: \quad c_{\beta} \mathcal{R}_{21}+s_{\beta} \mathcal{R}_{22}=c_{\alpha_{3}} s_{\beta-\alpha_{1}}-s_{\alpha_{2}} s_{\alpha_{3}} c_{\beta-\alpha_{1}}$

$g_{h_{3} V V}: \quad c_{\beta} \mathcal{R}_{31}+s_{\beta} \mathcal{R}_{32}=-s_{\alpha_{3}} s_{\beta-\alpha_{1}}-s_{\alpha_{2}} c_{\alpha_{3}} c_{\beta-\alpha_{1}}$,

which satisfy the following sum rule:

$\sum_{i=1}^{3} g_{h_{i} V V}^{2}=1$

This sum rule imply that each coupling $g_{h_{i} V V}$ is requested to satisfy: $\left|g_{h_{i} V V}\right| \leq 1$.

For the couplings between two Higgs bosons and one gauge boson, we can distinguish two cases, a neutral case which corresponds to $h_{i} A Z$ vertex and charged case associated with $h_{i} H^{\mp} W^{ \pm}$vertex. From the kinetic terms of the Higgs fields, one can derive the various trilinear couplings among neutral, charged Higgses and gauge bosons. In units of $\lambda_{n}=\frac{\sqrt{g^{2}+g^{\prime 2}}}{2}\left(p_{h_{i}}-p_{A}\right)_{\mu}$ for neutral Higgs, respectively in units of $\lambda_{c}=\mp \frac{g}{2}\left(p_{h_{i}}-p_{H^{ \pm}}\right)_{\mu}$ for charged Higgs, we have:

$g_{h_{1} V S}:-c_{\alpha_{2}} s_{\beta-\alpha_{1}}$, $g_{h_{2} V S}: c_{\alpha_{3}} c_{\beta-\alpha_{1}}+s_{\alpha_{2}} s_{\alpha_{3}} s_{\beta-\alpha_{1}}$,

$g_{h_{3} V S}:-s_{\alpha_{3}} c_{\beta-\alpha_{1}}+s_{\alpha_{2}} c_{\alpha_{3}} s_{\beta-\alpha_{1}}$,

where $\mathrm{V}=\mathrm{Z}$ and $S=A$ for the neutral case and $V=W^{ \pm}$ and $S=H^{\mp}$ for charged one.

In the N2HDM, one can easily derive the following sum rules:

$$
\begin{aligned}
& g_{h_{i} W^{ \pm} W^{\mp}}^{2}+g_{h_{i} W^{ \pm} H^{\mp}}^{2}+R_{i 3}^{2}=1, \quad i=1,2,3 \\
& g_{h_{i} Z Z}^{2}+g_{h_{i} Z A}^{2}+R_{i 3}^{2}=1, \quad i=1,2,3,
\end{aligned}
$$

where $R_{i 3}$ is the singlet component of the Higgs $h_{i}$.

There exist an other relation, which relates $h_{i} f \bar{f}$ and $h_{i} V V$ couplings and is given by:

$$
\sum_{i=1}^{3} g_{h_{i} V V} g_{h_{i} f \bar{f}}=1
$$

where $g_{h_{i} V V}$ and $g_{h_{i} f \bar{f}}$ are the normalized couplings of $h_{i}$ to gauge bosons and fermions. The above relationship Eq. (31) can be derived from the Feynman rules and using the orthogonality of $R$ matrix.

From above, it follows that:

- if $h_{i}$ is pure singlet $\left(R_{i 3}^{2} \approx 1\right)$, then from Eqs. $(29,30)$ one has $g_{h_{i} W W}^{2}+g_{h_{i} W^{ \pm} H^{\mp}}^{2} \approx 0$ and $g_{h_{i} Z Z}^{2}+g_{h_{i} Z A}^{2} \approx 0$ which would imply that $h_{i} V V, h_{i} H^{ \pm} W^{\mp}$ and $h_{i} A Z$ must be very suppressed, and this will present a real challenge for the production and detection of such Higgs bosons.

- if $g_{h_{i} V V}=1$ which means that $h_{i} V V$ is full strength, then both singlet component $R_{i 3}$ as well as $g_{h_{i} S V}$ couplings must vanish. This scenario could happen only when $h_{i}$ have no singlet component.

- According to Eq. (25), if $g_{h_{i} V V}=1$ then $g_{h_{j} V V}=0$ for $j \neq i$. This would imply from Eq. (31) that the reduced coupling to fermions must satisfy $g_{h_{i} f \bar{f}}=1$.

\section{Theoretical and experimental constraints}

The Two Higgs Doublets Model plus a Singlet possesses a large freedom in the scalar sector, coming from the large number of free parameters of the scalar potential. In order to obtain a viable model, many theoretical and experimental constraints have to be imposed on the scalar potential like perturbative unitarity, vacuum stability, electroweak precision observables and constraints from Higgs data. In what follows, we will briefly describe these constraints.

\subsection{Boundedness from below (BFB) of the potential}

In order to ensure a stable vacuum, the scalar potential has to be bounded from below in any directions in the field space 
as the field strength becomes extremely large. At large field values, the scalar potential is fully dominated by quartic couplings on which the BFB will depend only.

At large field strength, the potential defined by Eq. (2) is generically dominated by the quartic terms:

$$
\begin{aligned}
V^{(4)} & \left(H_{1}, H_{2}, S\right) \\
= & \frac{\lambda_{1}}{2}\left(H_{1}^{\dagger} H_{1}\right)^{2} \\
& +\frac{\lambda_{2}}{2}\left(H_{2}^{\dagger} H_{2}\right)^{2}+\lambda_{3} H_{1}^{\dagger} H_{1} H_{2}^{\dagger} H_{2}+\lambda_{4} H_{1}^{\dagger} H_{2} H_{2}^{\dagger} H_{1} \\
& +\frac{\lambda_{5}}{2}\left[\left(H_{1}^{\dagger} H_{2}\right)^{2}+\left(H_{2}^{\dagger} H_{1}\right)^{2}\right] \\
& +\frac{1}{8} \lambda_{6} S^{4}+\frac{1}{2} \lambda_{7}\left(H_{1}^{\dagger} H_{1}\right) S^{2}+\frac{1}{2} \lambda_{8}\left(H_{2}^{\dagger} H_{2}\right) S^{2}
\end{aligned}
$$

The study of $V^{(4)}\left(H_{1}, H_{2}, S\right)$ will thus be sufficient to obtain the main constraints. The full BFB constraints read as

$$
\begin{aligned}
& \lambda_{1}, \lambda_{2}, \lambda_{6}>0, \quad \lambda_{3}+\sqrt{\lambda_{1} \lambda_{2}}>0 \\
& \lambda_{3}+\lambda_{4}-\left|\lambda_{5}\right|+\sqrt{\lambda_{1} \lambda_{2}}>0 \\
& \lambda_{7}>-\sqrt{\lambda_{1} \lambda_{6}}, \quad \lambda_{8}>-\sqrt{\lambda_{2} \lambda_{6}}
\end{aligned}
$$

for $\lambda_{7}>0$ and $\lambda_{8}>0$.

If $\lambda_{7}$ or $\lambda_{8}<0$, we have to satisfy two additional constraints:

$$
\begin{aligned}
& \lambda_{3} \lambda_{6}-\lambda_{7} \lambda_{8}+\sqrt{\left(\lambda_{1} \lambda_{6}-\lambda_{7}^{2}\right)\left(\lambda_{2} \lambda_{6}-\lambda_{8}^{2}\right)}>0 \\
& \lambda_{6}\left(\lambda_{3}+\lambda_{4}+\left|\lambda_{5}\right|\right)-\lambda_{7} \lambda_{8} \\
& \quad+\sqrt{\left(\lambda_{1} \lambda_{6}-\lambda_{7}^{2}\right)\left(\lambda_{2} \lambda_{6}-\lambda_{8}^{2}\right)}>0
\end{aligned}
$$

Full technical details on the proof of these constraints can be found in Appendix (A).

\subsection{Perturbative unitarity}

To constrain the scalar potential parameters of the N2HDM further one can ask that tree-level perturbative unitarity is preserved for a variety of scattering processes: gauge bosongauge boson scattering, scalar-scalar scattering and also scalar-gauge boson scattering. Since the equivalence theorem states that at high energy limit $\sqrt{s}$ the amplitudes of a scattering process involving longitudinally polarized gauge bosons $\mathrm{V}$ are asymptotically equal, up to correction of the order $m_{V} / \sqrt{s}$, to the corresponding scalar amplitudes in which longitudinally polarized gauge bosons are replaced by their corresponding Goldstone bosons. We conclude that perturbative unitarity constraints can be implemented by considering pure scalar-scalar scattering only.

In order to derive the perturbative unitarity constraints on the scalar parameters of N2HDM we follow Refs. [38,39]. According to [38,39], one computes the scattering amplitude in the weak eigenstate basis where the quartic couplings have only $\lambda_{i}$ dependence and no dependence on the mixing angles: $\alpha_{i}$ and $\beta$. The important point is that the amplitude expressed in the mass eigenstate fields can be transformed into the amplitude for the non-physical fields by making a unitary transformation. The eigenvalues for the scattering amplitude should be unchanged under such a unitary transformation.

In the Appendix (B) we present the technical details of the different $2 \rightarrow 2$ scattering amplitudes. The explicit forms of the eigenvalues at tree level are given by:

$$
\begin{array}{r}
\left|\lambda_{3}+\lambda_{4}\right|,\left|\lambda_{3} \pm \lambda_{5}\right|,\left|\lambda_{3}+2 \lambda_{4} \pm 3 \lambda_{5}\right|<8 \pi \\
\left|\frac{\lambda_{7}}{2}\right|,\left|\frac{\lambda_{8}}{2}\right|,\left|\frac{\lambda_{6}}{4}\right|<8 \pi \\
\left|\frac{1}{2}\left(\lambda_{1}+\lambda_{2} \pm \sqrt{\left(\lambda_{1}-\lambda_{2}\right)^{2}+4 \lambda_{4}^{2}}\right)\right|<8 \pi \\
\left|\frac{1}{2}\left(\lambda_{1}+\lambda_{2} \pm \sqrt{\left(\lambda_{1}-\lambda_{2}\right)^{2}+4 \lambda_{5}^{2}}\right)\right|<8 \pi
\end{array}
$$

Other eigenvalues are coming from the cubic polynomial equation associated to the submatrix $\mathcal{M}_{2}$ corresponds to scattering with one of the following initial and final states: $\left(\phi_{1}^{+} \phi_{1}^{-}, \phi_{2}^{+} \phi_{2}^{-}, \frac{\phi_{1} \phi_{1}}{\sqrt{2}}, \frac{\phi_{2} \phi_{2}}{\sqrt{2}}, \frac{\phi_{s} \phi_{s}}{\sqrt{2}}, \frac{\chi_{1} \chi_{1}}{\sqrt{2}}, \frac{\chi_{2} \chi_{2}}{\sqrt{2}}\right)$. For more details, see Appendix (B).

Moreover, we also force the potential to be perturbative by imposing that all quartic couplings of the scalar potential satisfy $\left|\lambda_{i}\right| \leq 8 \pi(i=1, \ldots, 8)$.

\subsection{Electroweak precision test observables (EWPT)}

The oblique parameters $\mathrm{S}, \mathrm{T}$, and $\mathrm{U}$ are known to provide an indirect probe of new physics BSM for theories that process $S U(2) \times U(1)$ symmetry $[41,42]$. These parameters quantify deviations from the SM in terms of radiative corrections to the $\mathrm{W}, \mathrm{Z}$ and the photon self-energies. In the framework of N2HDM, the Higgs doublet couples to the $\mathrm{W}$ and $\mathrm{Z}$ gauge bosons via the covariant derivative. Due to singlet and doublet admixtures in the scalar sector, the singlet field will also couple to the gauge bosons $\mathrm{W}$ and $\mathrm{Z}$. Therefore, both neutral Higgs $h_{i}, A$ and charged Higgs will contribute to $\mathrm{S}$ and T parameters which are very well constrained by electroweak precision test observables. These EWPT constraints will be converted to limits on the mixing angles and/or masses splitting among the N2HDM spectrum. The extra-contribution to $\mathrm{S}, \mathrm{T}$ and $\mathrm{U}$ parameters for $\mathrm{N} 2 \mathrm{HDM}$ are given in Appendix (C).

In order to study the correlation between $\mathrm{S}$ and $\mathrm{T}$, we perform the $\chi^{2}$ test over the allowed parameter space of N2HDM. Our $\chi_{S, T}^{2}$ is defined as:

$$
\begin{aligned}
\chi_{S, T}^{2}= & \frac{1}{\hat{\sigma}_{1}^{2}\left(1-\rho^{2}\right)}(S-\hat{S})^{2}+\frac{1}{\hat{\sigma}_{2}^{2}\left(1-\rho^{2}\right)}(T-\hat{T})^{2} \\
& -\frac{2 \rho}{\hat{\sigma}_{1} \hat{\sigma}_{2}\left(1-\rho^{2}\right)}(S-\hat{S})(T-\hat{T}),
\end{aligned}
$$


where $\mathrm{S}$ and $\mathrm{T}$ are the computed quantities within N2HDM framework $[22,43,44] . \hat{S}$ and $\hat{T}$ are the measured values of $S$ and $\mathrm{T}, \hat{\sigma}_{1,2}$ are their one-sigma errors and $\rho$ their correlation [45],

$S=0.04 \pm 0.11, \quad T=0.09 \pm 0.14, \quad \rho_{S, T}=0.92$

It is worth noting here that we have checked the limits on the oblique parameters with the $2 \mathrm{HDM}$, in this sense, our results match exactly to those outlined in $[46,47]$.

In addition, we have indirect experimental constraints from $B$ physics observables on the contribution of the N2HDM such as $\tan \beta$ and $m_{H^{ \pm}}$. In the N2HDM, the charged Higgs coupling to fermions is not at all affected by the singlet component of the additional Higgs. Therefore, constraints from $B \rightarrow X_{s} \gamma$ and $B_{q}$ mixing will be the same as for the usual 2HDM model. We remind the reader that the recent experimental results presented by the Heavy Flavor Averaging Group (HFAG) [48] of $B\left(B \rightarrow X_{s} \gamma\right)$ have changed in a significant way the bounds on the charged Higgs boson mass. For instance, in N2HDM Type-II, the measurement of the $\mathrm{BR}\left(B \rightarrow X_{s} \gamma\right)$ constrains charged Higgs mass to be larger than about $570 \mathrm{GeV}[45,49]$, while in Type-I one can still obtain a charged Higgs boson with a mass as low as $100-200 \mathrm{GeV}$ provided that $\tan \beta \geq 2$. In addition, recent analysis [45] for the 2HDM shows that $\Delta m_{s}\left(\operatorname{resp} B_{d} \rightarrow \mu \mu\right)$ constraint requests that $\tan \beta>2.5$ (resp $\tan \beta>3$ ). In fact, $\Delta m_{s}$ is only sensitive to charged Higgs, then the limit $\tan \beta>2.5$ would apply both for 2HDM and N2HDM. However, $B_{d} \rightarrow \mu \mu$ is sensitive both to charged Higgs as well as to neutral higgses $h_{1,2,3}$, therefore the limit on $\tan \beta$ could be different in 2HDM and N2HDM. Therefore, in what follows we will require that $\tan \beta>2.5$.

\subsection{Constraints from Higgs data}

Both ATLAS and CMS experiments of the LHC Run1 with 7 and $8 \mathrm{TeV}$ and Run 2 with $13 \mathrm{TeV}$ confirmed the discovery of a Higgs boson with a mass around $125 \mathrm{GeV}$. Both groups performed several measurements on the Higgs boson couplings to the SM particles. Recently, both ATLAS and CMS Collaborations have announced the observation of Higgs bosons produced together with a top-quark pair $[8,9]$. All these measurements seem to be in perfect agreement with SM predictions.

In the case of N2HDM, all tree-level Higgs couplings to fermions and gauge bosons are modified with the mixing parameters $\alpha_{i}$ and $\beta$. The loop-mediated processes such as $g g \rightarrow h_{i}, h_{i} \rightarrow \gamma \gamma$ and $h_{i} \rightarrow \gamma Z$ will be affected both by the mixing angles as well as by the additional charged Higgs $H^{ \pm}$loops which depend on the triple scalar coupling $h_{i} H^{ \pm} H^{\mp}$.

To study the effects of ATLAS and CMS measurements on N2HDM, we take into account experimental data from the observed cross section times branching ratio divided by the corresponding SM predictions for the various channels, i.e. the signal strengths of the Higgs boson defined by:

$\mu_{i}^{f}=\frac{\sigma_{i}(h) B r(h \rightarrow f)}{\sigma_{i}^{S M}(h) B r^{S M}(h \rightarrow f)}$

where $i$ stand for different modes of Higgs production. The dominant mechanisms of Higgs production are gluon fusion ( $g g F)$, followed by vector boson fusion (VBF), Higgsstrahlung (Vh) and associated production with top-quark pairs $(\bar{t} t h)$.

All these various signal strength channels are included in our analysis through the public code HiggsBounds [50-52] and HiggsSignals [53] which also include previous LEP and Tevatron experimental searches.

As said previously, in our analysis, we will assume that $h_{2}$ is the $125 \mathrm{GeV}$ Higgs boson discovered while $h_{1}$ would be lighter than $h_{2}$. Therefore, once the decay channels $h_{2} \rightarrow$ $h_{1} h_{1}$ and/or $h_{2} \rightarrow A A$ are open, the subsequent decays of $h_{1} / A$ into fermions, photons or gluons, will lead either to invisible or undetected $h_{2}$ decays that can be constrained by using global analysis to the present ATLAS and CMS data to Higgs couplings.

We stress here that there are also searches for non-detected decays of the SM Higgs boson both by ATLAS and CMS. CMS looks for the following SM Higgs production channels: gluon fusion, vector boson fusion, and Higgsstrahlung process $p p \rightarrow V H(\mathrm{~V}=\mathrm{W}$ or $\mathrm{Z})$ with subsequent invisible Higgs decays. Upper limits are placed on $\mathrm{Br}(H \rightarrow$ invisible $)$, as a function of the assumed production cross sections. The combination of all the above channels, assuming SM production, yields an upper limit of 0.24 on the $B R(H \rightarrow$ invisible $)$ at the $95 \%$ confidence level [54]. ATLAS collaboration performs a search for an invisible decay of the Higgs through $p p \rightarrow Z H$ process with a leptonic subsequent decay of the $\mathrm{Z}$ [55]. Their limit is slightly weaker than CMS results. In addition, it's worth mentioning that a new observed (expected) upper limit of $0.19(0.15)$ has been derived recently on the $B R(H \rightarrow$ invisible $)$ at the $95 \%$ confidence level [56] from the combination of $\sqrt{s}=7,8$ and $13 \mathrm{TeV}$ searches data.

In our study, we will use the fact that the total branching fraction of the SM-like Higgs boson into undetected BSM decay modes is constrained, as mentioned, by $B R(H \rightarrow$ invisible $) \leq 0.24$ where $B R(H \rightarrow$ invisible $)$ designate $B R\left(h_{2} \rightarrow h_{1} h_{1}\right)$ or the sum of $B R\left(h_{2} \rightarrow h_{1} h_{1}\right)$ and $B R\left(h_{2} \rightarrow A A\right), B R\left(h_{2} \rightarrow Z^{*} A\right)$ and $B R\left(h_{2} \rightarrow W^{*} H^{ \pm}\right)$ if the later is open. ${ }^{1}$

\footnotetext{
${ }^{1}$ Using 0.24 as upper limit instead of the recent one 0.19 will not affect the result too much since the $\operatorname{Br}\left(h_{2} \rightarrow h_{1} h_{1}\right)$ never exceed 0.2 value (see Fig. 8). Also, several searches have appeared recently which might further constrain the model's parameter space [57,58].
} 


\section{Numerical results}

Before discussing our results, we would like to comment on previous related works. In the previous studies on the N2HDM, several phenomenologically viable scenarios of N2HDM type-I and type-II were summarized in Ref. [31,59, $60]$. These studies discuss the allowed singlet-doublet admixture of Higgses $h_{i}$ and also analyze the production and the decay rate of the non SM-like Higgs bosons into the most important SM final state channels, where one of the neutral scalar $h_{i}$ is chosen as the discovered Higgs boson at $125 \mathrm{GeV}$, $h_{125}$. The mass-degenerate case is suppressed by requiring a $5 \mathrm{GeV}$ mass splitting between the $h_{125}$ and the other neutral scalar.

In the above-mentioned studies, the branching ratios and decay widths of the Higgs bosons of the Next-to-TwoHiggs-Doublet Model (N2HDM) are computed with the N2HDECAY ${ }^{2}$ code. The program is documented in [61].

We recall that another recent study on the extension of $2 \mathrm{HDM}$ by adding a singlet scalar is presented in Ref. [62], which discusses the implication of this extension on the decay rate of the heavy pseudo-scalar and the charged Higgs boson.

As mentioned previously, we concentrate here on the case where $h_{2}$ is the SM-like Higgs, $h_{1}$ is lighter than $125 \mathrm{GeV}$ and investigate the phenomenology of the neutral Higgses $h_{1,2,3}$.

\subsection{Parameter scan}

The scalar potential Eq. (2) has 15 independent parameters: four masses, 8 quartic couplings $\lambda_{1, \ldots, 8}$ and 3 vacuum expectation values. Three masses can be eliminated by the use of the 3 minimization conditions Eq. (6). Moreover, after electroweak symmetry breaking, from the kinetic terms of the Higgs doublets, the $\mathrm{W}$ and $\mathrm{Z}$ gauge bosons acquire masses which are given by $m_{W}^{2}=\frac{1}{2} g^{2}\left(v_{1}^{2}+v_{2}^{2}\right)$ and $m_{Z}^{2}=\frac{1}{2}\left(g^{2}+g^{\prime 2}\right)\left(v_{1}^{2}+v_{2}^{2}\right)$, where $g$ and $g^{\prime}$ are the $S U_{L}(2)$ and $U_{Y}(1)$ gauge couplings. The combination $v_{1}^{2}+v_{2}^{2}$ is thus fixed by the electroweak scale through the well known relation $v^{2}=v_{1}^{2}+v_{2}^{2}=\left(2 \sqrt{2} G_{F}\right)^{-1}$, and we are left with 11 free parameters. By simple algebraic calculations, from the mass matrix relations, one can express all the quartic couplings $\lambda_{i}$ as a function of the physical masses, $\mu^{2}, \tan \beta$ and the mixing angles $\alpha_{i} \cdot{ }^{3}$ One can then take the following set of free independent parameters:

\footnotetext{
2 The implemented decay widths include the most important state-ofthe-art higher order QCD corrections and the important off-hell decays [31,61].

${ }^{3}$ In Ref. [31] one can find the expressions of all $\lambda_{i} \mathrm{~s}$ as well as the trilinear and quartic scalar couplings as a function of the physical masses and the mixing angles (see Table 1).
}

$\alpha_{1,2,3}, \tan \beta, v_{S}, m_{h_{1,2,3}}, m_{A}, m_{H^{ \pm}}$and $\mu^{2}$

with the convention $m_{h_{1}}<m_{h_{2}}<m_{h_{3}}$.

Note that the usual $2 \mathrm{HDM}$ is recovered from N2HDM by taking the following limits:

$$
\alpha_{1} \rightarrow \alpha+\frac{\pi}{2}, \quad \alpha_{2} \rightarrow 0 \text { and } \alpha_{3} \rightarrow 0
$$

In our analysis, we will study the consistency of having the second Higgs $h_{2}$ as the $125 \mathrm{GeV}$ SM-like Higgs. This scenario opens morebeyond Standard Model decay channels for $h_{2}$ such as $h_{2} \rightarrow h_{1} h_{1}$ and probably $h_{2} \rightarrow$ $A A, A A^{*}, Z^{*} A, W^{*} H^{ \pm}$. In order to calculate the decay widths and branching fractions of the Higgs bosons, we have implemented a private Fortran code, based on HDECAY [63]. The main program is linked to HiggsBounds and HiggsSignals libraries. We also implement the theoretical constraints (unitarity and boundedness from below), the oblique parameters (S, T, U). In the computation of the decay width of $h_{i}$, we include the off-shell decays such as $h_{i} \rightarrow\left\{Z^{*} A, W^{* \mp} H^{ \pm}\right\}$ and $h_{i} \rightarrow V V^{*}$ and also $h_{i} \rightarrow V^{*} V^{*}$ if needed. We note also that in the case of $h_{i} \rightarrow \gamma V$, with $V=\gamma, Z$, we include $\mathrm{W}$, top, bottom and charged Higgs loops together with the high order QCD corrections to $h_{i} \rightarrow \gamma V$.

In order to display the allowed regions for the parameters, we have considered both of the exclusions from both HiggsBounds-5.1beta and HiggsSignals-2.1.0beta to compute the value of $\chi_{\min }^{2}$ considering the combination of $8 \mathrm{TeV}$ and $13 \mathrm{TeV}$ Higgs signal strength data from run-I and run-II. Thus, we show the best fit at $95.5 \% \mathrm{CL}$, which corresponds to $\Delta \chi^{2} \leq 5.99$ where $\Delta \chi^{2}=\chi^{2}-\chi_{\text {min }}^{2}$.

In the left panel of Fig. 1, we show the allowed parameter for $\lambda_{7,8}$ for various values of $\lambda_{6}$ taking into account perturbative unitarity and BFB constraints. We note first that there is a complete overlap between the three colors for positive $\lambda_{7,8}$. One can see that for negative $\lambda_{7,8}$, the theoretical constraints restrict their strength for small $\lambda_{6}$. The restriction is relaxed for large $\lambda_{6} \approx 4 \pi$.

In the right panel of Fig. 1, we present the correlation between $\mathrm{S}$ and $\mathrm{T}$, after taking into account the theoretical constraints and the exclusion from Higgs Bounds at 95\% $\mathrm{CL}$. The red, green and black points represent the points with $\Delta \chi_{S T}^{2}$ value within the $1 \sigma, 2 \sigma$ and $3 \sigma$ interval. Note that $\Delta \chi_{S T}^{2}=\chi_{S T}^{2}-\chi_{S T}^{2, \min }$ where $\chi_{S T}^{2, \min }$ is the minimum value of $\chi_{S T}^{2}$ given by Eq. (38).

\subsection{Results for N2HDM type I}

In this section, we study the case of N2HDM type I, where both $h_{1}$ and $A$ could be rather light. We scan over the following range:

$$
\begin{aligned}
& m_{h_{1}} \in[10,120] \mathrm{GeV}, \quad m_{h_{3}} \in[200,700] \mathrm{GeV}, \\
& m_{H^{ \pm}} \in[80,700] \mathrm{GeV},
\end{aligned}
$$


Fig. 1 The left panel shows the parameter space allowed by the theoretical constraints (Unitarity, Perturbativity and BFB) for $\left(\lambda_{7}, \lambda_{8}\right)$ for $\lambda_{6}=(1,4,12)$. Note the complete overlap between black, black and red for positive $\lambda_{6,7}$. The right panel illustrates the correlation between oblique parameters $\mathrm{S}$ and $\mathrm{T}$. The errors for $\chi_{S T}^{2}$-square fit are $99.7 \% \mathrm{CL}$ (black), $95.5 \% \mathrm{CL}$ (green) and $68 \%$ CL (red)
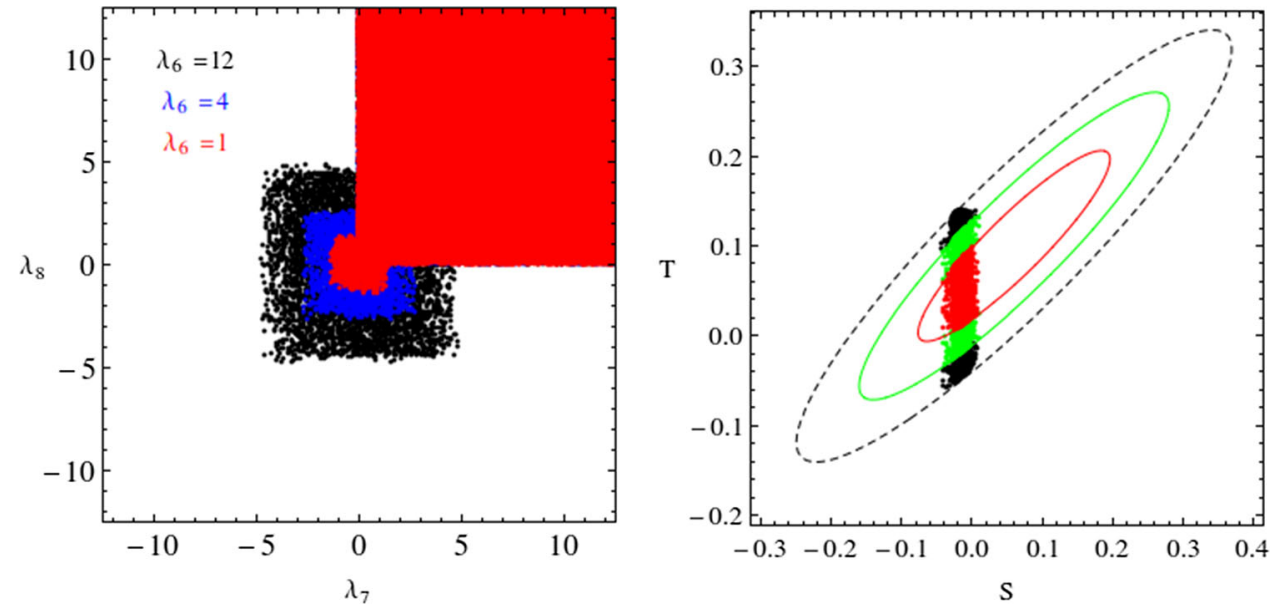

$$
\begin{aligned}
& m_{A} \in[62.5,700] \mathrm{GeV}, \quad \mu^{2} \in\left[0,1.5 \times 10^{3}\right] \mathrm{GeV}, \\
& v_{S}=300 \mathrm{GeV}, \\
& \frac{-\pi}{2}<\alpha_{1}<\frac{\pi}{2}, \quad \frac{-\pi}{6}<\alpha_{2,3}<\frac{\pi}{6}, \quad \text { and } 2.5<\tan \beta<25,
\end{aligned}
$$

In our scan we allow $h_{1}$ to be in the range $[10,124]$ $\mathrm{GeV}$ while $m_{A} \geq 62.5 \mathrm{GeV}$. In such configuration, only $h_{2} \rightarrow h_{1} h_{1}$ and/or $h_{2} \rightarrow A Z^{*}, H^{ \pm} W^{\mp *}$ can be open. $h_{2} \rightarrow A A^{*} \rightarrow A f f^{\prime}$ is suppressed both by the phase space and the coupling of $A$ to light fermions. The non detected decays of $h_{2}$, which is identified here as the Higgs-SM-like, such as $h_{2} \rightarrow h_{1} h_{1}, A A^{*}, A Z^{*}, H^{ \pm} W^{\mp *}$ if open should not exceed $24 \%$ as we explain below.

To be consistent with the EW precision measurements, such light $h_{1}$ and A are naturally also accompanied by a light charged Higgs. We recall that a light charged Higgs state is allowed by the constraint $B \rightarrow X_{s} \gamma$ in N2HDM of type I.

$h_{1}$ decays

We study first the decay of $h_{1}$ into SM particles. As one can see from the couplings of $h_{1}$ to fermions given by Eq. (19), $h_{1}$ could be fermiophobic if $R_{12} \propto \cos \alpha_{2} \sin \alpha_{1}$ vanish. This scenario might happen if we take $\alpha_{1} \approx 0$ and/or $\alpha_{2} \approx \pi / 2$ which is possible since both $\alpha_{1}$ and $\alpha_{2}$ are free parameters in this model.

- The case where $\alpha_{2}=\pi / 2$ corresponds to $h_{1}$ being pure singlet and will not be discussed here.

- The case where $\alpha_{1}=0$ with $\alpha_{2} \neq \pi / 2, h_{1}$ contains both doublet and singlet component.

In Fig. 2, we show the branching ratio of $h_{1} \rightarrow W^{+} W^{-}$ as a function of $h_{1} \rightarrow \gamma \gamma$ with $\operatorname{Br}\left(h_{1} \rightarrow b \bar{b}+\tau^{+} \tau^{-}\right)$ represented on the horizontal axis on the left panel, while on the right panel, we show $m_{h_{1}}$. Since $m_{h_{1}} \leq 125 \mathrm{GeV}$, $h_{1} \rightarrow W^{+} W^{-}$will proceed with one or both W being offshell. We first mention that the singlet component of $h_{1}$ does not exceed $50 \%$ in our case, which makes $h_{1}$ dominated by doublet components. $h_{1}$ has a large doublet component due to the large uncertainties on the LHC measurement, which does not constraint too much $h_{2} V V$ and $h_{2} f f$ couplings to be fully SM-like, and this leaves rather large room for $h_{1} V V$ as well as $h_{3} V V$. Fitting $h_{1} V V$ within LHC uncertainties still allows $h_{1}$ to have large doublet component.

As one can see, in most cases $h_{1}$ would decay significantly into a bottom pair unless $\alpha_{1}$ vanishes, which is the fermiophobic limit for $h_{1}$. In this case, it is clear that $h_{1} \rightarrow \gamma \gamma$ could reach its maximum value, when $\operatorname{Br}\left(h_{1} \rightarrow b \bar{b}\right)$ and $\operatorname{Br}\left(h_{1} \rightarrow \tau^{+} \tau^{-}\right)$are very suppressed. When $h_{1}$ is fermiophobic, $h_{1} \rightarrow V V^{*}$ or $h_{1} \rightarrow V^{*} V^{*}, V=Z, W$ can compete with $h_{1} \rightarrow \gamma \gamma$. In what follows, we only discuss $h_{1} \rightarrow W W$ since $h_{1} \rightarrow Z Z$ is smaller. In fact, $h_{1} \rightarrow W^{*} W^{*}$ which is open for $m_{h_{1}}<m_{W}$ is very suppressed due to phase space while for $m_{h_{1}} \geq m_{W}, h_{1} \rightarrow W W^{*}$ is open and could strongly compete with $h_{1} \rightarrow \gamma \gamma$. This is shown in the left and right panel of Fig. 2, where we can see $\operatorname{Br}\left(h_{1} \rightarrow W W^{*}\right)$ as a function of $\operatorname{Br}\left(h_{1} \rightarrow \gamma \gamma\right)$. Close to the fermiophobic limit where $h_{1} \rightarrow b \bar{b}$ and $h_{1} \rightarrow \tau^{+} \tau^{-}$are suppressed, if the mass of $h_{1}$ is larger than the $\mathrm{W}$ boson mass, then $h_{1} \rightarrow W W^{*}$ can dominate $h_{1} \rightarrow \gamma \gamma$ in some cases.

One could have also the following scenario: both $h_{1} \rightarrow$ $f \bar{f}, h_{1} \rightarrow V V^{*}$ and $h_{1} \rightarrow \gamma \gamma, \gamma Z$ are rather small, while the branching ratio of $\operatorname{Br}\left(h_{1} \rightarrow A Z^{*}\right)+\operatorname{Br}\left(h_{1} \rightarrow\right.$ $H^{ \pm} W^{* \mp}$ ) becomes significant which can be understood from the sum rules Eq. (30). Due to the smallness of $h_{1} V V$ coupling and $R_{13}$ component, the sum rule given by Eq. (30) implies that $h_{1} A Z$ and $h_{1} W^{ \pm} H^{\mp}$ could become significant. This is illustrated in the left panel of Fig. 2 with the black dots in the left-down corner where both $\operatorname{Br}\left(h_{1} \rightarrow W W^{*}\right) \approx$ $B r\left(h_{1} \rightarrow \gamma \gamma\right) \approx 10^{-4}$ and also $\sum B r\left(h_{1} \rightarrow \tau^{+} \tau^{-}+b \bar{b}\right)$ are rather small. 

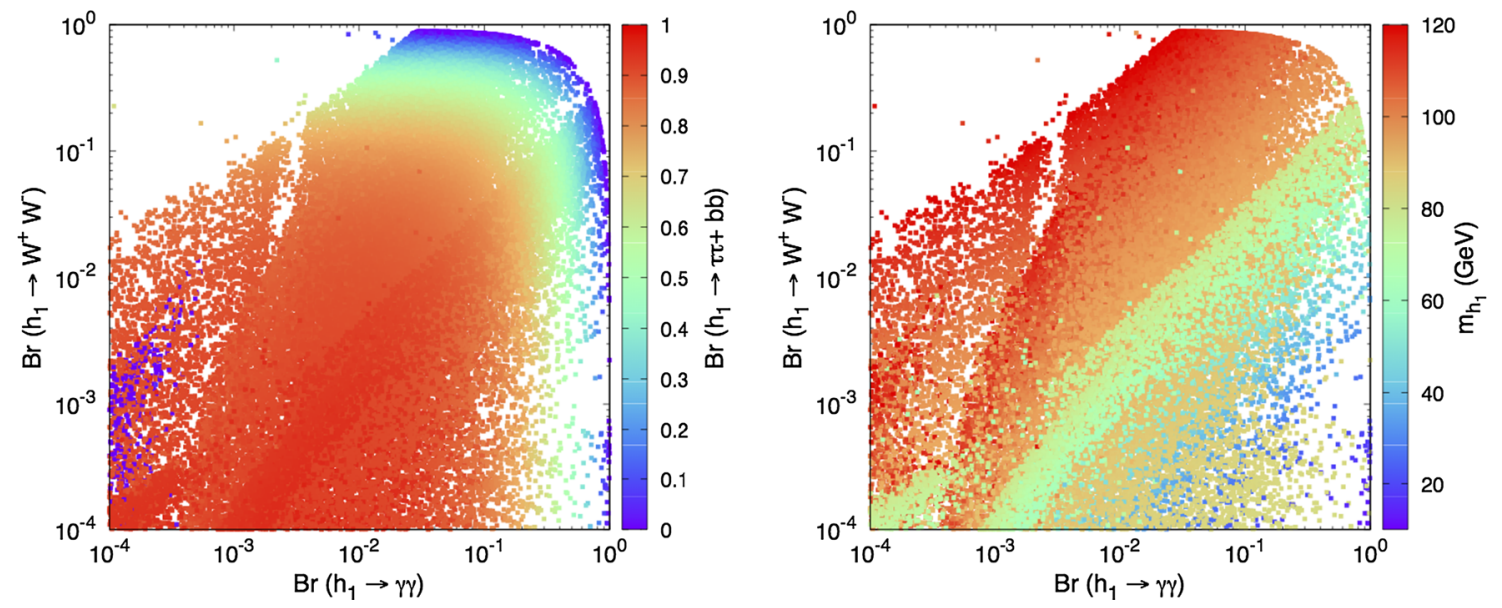

Fig. $2 \operatorname{Br}\left(h_{1} \rightarrow W^{+} W^{-}\right)$and $\operatorname{Br}\left(h_{1} \rightarrow \gamma \gamma\right)$ vs. $\operatorname{Br}\left(h_{1} \rightarrow b \bar{b}, \tau^{+} \tau^{-}\right)$(left), and $m_{h_{1}}$ (right) at $95 \% . C L$
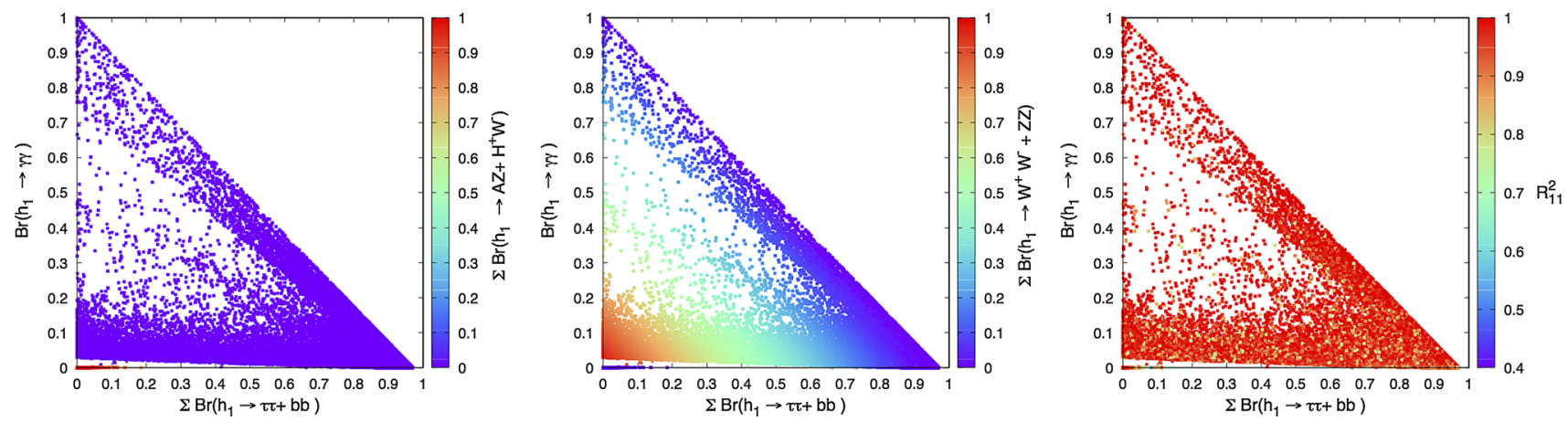

Fig. $3 \mathrm{Br}\left(h_{1} \rightarrow \gamma \gamma\right)$ as a function of $\operatorname{Br}\left(h_{1} \rightarrow \tau^{+} \tau^{-}+b \bar{b}\right)$ vs. $\operatorname{Br}\left(h_{1} \rightarrow\left(H^{ \pm} W^{\mp}+A Z\right)\right.$ (left panel), $B r\left(h_{1} \rightarrow W^{+} W^{-}\right)$(middle panel) and $R_{11}^{2}$ (right panel) at $95 \%$ C.L

The above configuration is illustrated clearly in Fig. 3 (left and middle) where we show the correlation between $\sum \operatorname{Br}\left(h_{1} \rightarrow \tau^{+} \tau^{-}+b \bar{b}\right)$ and $\operatorname{Br}\left(h_{1} \rightarrow \gamma \gamma\right)$ as a function of $\operatorname{Br}\left(h_{1} \rightarrow H^{ \pm} W^{\mp}+A Z\right)$ and $\operatorname{Br}\left(h_{1} \rightarrow W^{+} W^{-}\right)$on the horizontal axis. It can be seen that, when the fermionic $\left(\tau^{+} \tau^{-}, b \bar{b}\right)$ and bosonic $\left(\gamma \gamma, W^{+} W^{-}, \mathrm{ZZ}\right)$ decays of $h_{1}$ are suppressed, the Higgs to Higgs decays $h_{1} \rightarrow H^{ \pm} W^{\mp}$ and/or $h_{1} \rightarrow Z A$ become significant. As it can be seen, this happens only in a tiny region of the parameter space. In the right panel of Fig. 3, we can see that $h_{1}$ has a large doublet component in most cases. The fact that $\operatorname{Br}\left(h_{1} \rightarrow H^{ \pm} W^{\mp}\right)$ and/or $\operatorname{Br}\left(h_{1} \rightarrow Z A\right)$ are either maximal $100 \%$ or minimal close to $0 \%$ with no intermediate range is mainly due to HiggsSignal which requires that $\chi^{2}$ should have a correct value.

In Fig. 4 we illustrate $\kappa_{f}^{h_{1}}$ as a function of $\kappa_{V}^{h_{1}}$ with $R_{1 i}(i=$ $1,2,3)$ component of $h_{1}$ on the horizontal axis. Note that $\kappa_{V}^{h_{i}}$ and $\kappa_{f}^{h_{i}}$ are the normalized couplings of neutral Higgs to a pair of gauge bosons and to a pair of fermions respectively, discussed in Sects. (2.3) and (2.4); $\kappa_{V}^{h_{i}} \equiv g_{h_{i} V V}$ and $\kappa_{f}^{h_{i}} \equiv$ $g_{h_{i} f f}$.
From this plot, one can read that the doublet component is rather large in most of the cases leaving only small singlet component which is less than 50\%. One can also learn that when $\kappa_{f}^{h_{1}}$ and $\kappa_{V}^{h_{1}}$ are suppressed, the doublet component is very large. Which means that $h_{1}$ is mainly coming from the doublet components. According to the sum rule Eq. (25), the strength of the SM Higgs interaction, at tree level, is shared by the three neutral Higgs bosons of the N2HDM, thus, the couplings of the neutral scalars to vector bosons cannot be enhanced over the SM value and for that we have $\left|\kappa_{V}^{h_{1}}\right| \leq 1$ which is consistent with Fig. 4. On the other hand, there is a large area of the parameter space where $\left|\kappa_{f}\left(h_{1}\right)\right| \leq 1$. On the right panel of Fig. 4, we show the sensitivity to $\tan \beta$ where we can see a linear correlation between $\kappa_{f}^{h_{1}}$ and $\kappa_{V}^{h_{1}}$ at large $\tan \beta$.

Let us mention that in this scenario with suppressed $h_{1} f \bar{f}$ and $h_{1} V V$ couplings, $h_{1}$ can not be produced in the usual channel such as gluon fusion, vector boson fusion or Higgsstrahlung. According to sum rules Eq. (30), if the singlet component of $h_{1}$ is small and $h_{1} V V$ coupling is suppressed, then $h_{1} Z A$ and $h_{1} W^{ \pm} H^{\mp}$ are enhanced, therefore $h_{1}$ can be produced in one of the following processes: $p p \rightarrow Z^{*} \rightarrow$ 

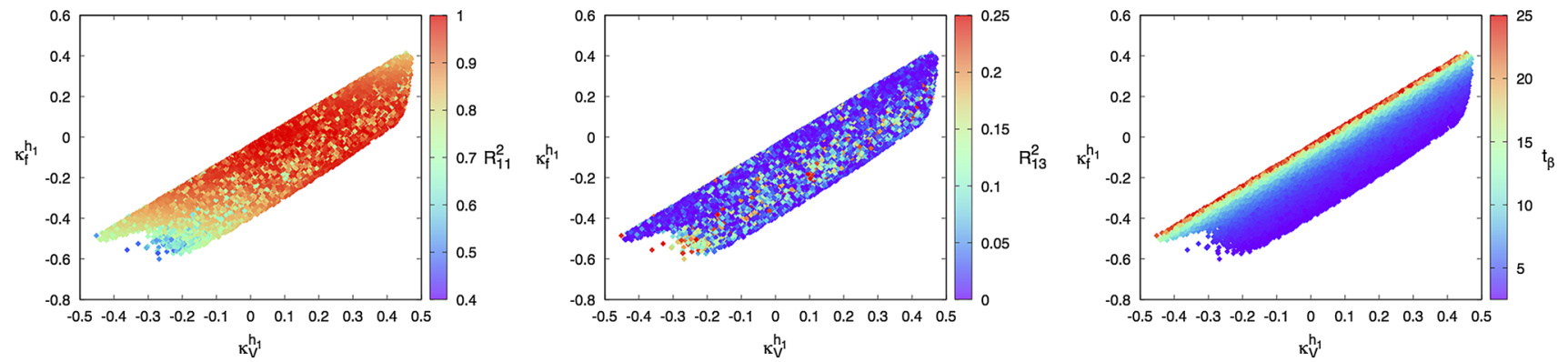

Fig. $4 \kappa_{f}^{h_{1}}$ as a function of $\kappa_{V}^{h_{1}}$ with $R_{1 i}^{2}$ (i=1,3) on the horizontal axis (left and middle panel), and with tan $\beta$ on the right panel
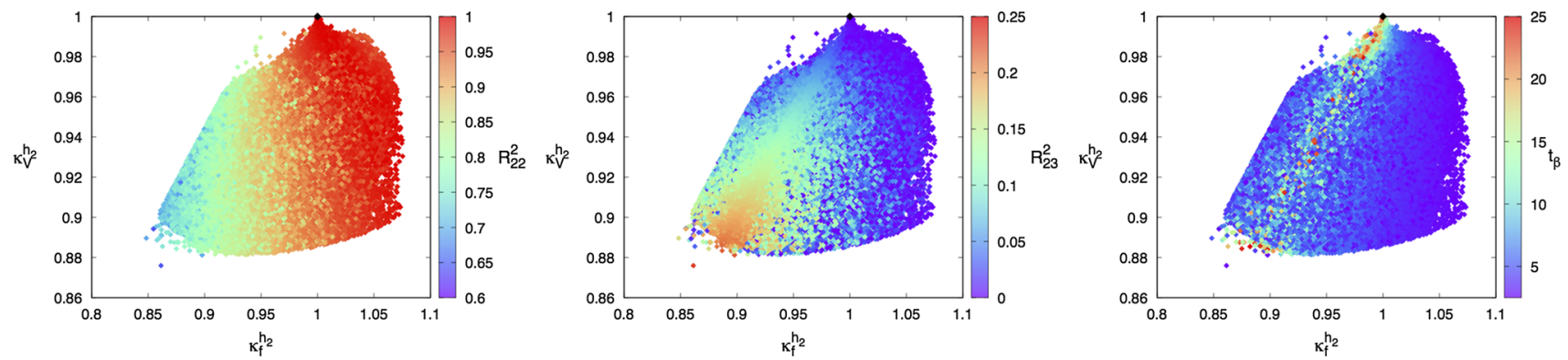

Fig. $5\left(\kappa_{V}^{h_{2}}, \kappa_{f}^{h_{2}}\right)$ in N2HDM type-I as a function of $R_{22,23}$ (left and middle panel), and $\tan \beta$ on the right panel. The black lozenge stands for the SM value

$h_{1} A$ or $p p \rightarrow W^{*} \rightarrow h_{1} H^{ \pm}$which would lead respectively to the following final states $Z A A$ or $W H^{ \pm} H^{\mp}$.

$h_{2}$ decays

We now discuss the decay of the SM-like $h_{2}$. We first show the consistency of $h_{2} \rightarrow V V$ and $h_{2} \rightarrow f \bar{f}$ with LHC data. For this purpose, we illustrate in Fig. 5 the correlation between $\kappa_{V}^{h_{2}}$ and $\kappa_{f}^{h_{2}}$ as a function of $R_{2 i}^{2}$. According to the sum rule Eq. (25), $\kappa_{V}^{h_{2}}<1$, and this is clearly illustrated in the plot. One can see from the plot that when $\kappa_{V}^{h_{2}} \approx 1$ we have also $\kappa_{f}^{h_{2}} \approx 1$, this is a consequence of the sum rule Eq. (31). However, the suppression of $\kappa_{V}^{h_{2}}$ could be of the order of $12 \%$ and it could happen both for $\kappa_{f}^{h_{2}}<1$ or $\kappa_{f}^{h_{2}}>1$. Note that the suppression of both $\kappa_{f}^{h_{2}}$ and $\kappa_{V}^{h_{2}}$ takes place when the singlet component of $h_{2}$ is rather large $R_{23}^{2}>0.1$. One can see that $\kappa_{f}^{h_{2}}$ could reach a value less than 0.8 for $R_{23}^{2} \approx 0.25$. It is also clear from the plot that one can have an enhancement of $\kappa_{f}^{h_{2}}$ in the range of [1.05-1.15] for small singlet component of $h_{2}\left(R_{23}^{2} \approx 0.1\right)$ and moderate $\tan \beta$.

In Fig. 6, we show the correlation between $\kappa_{g g}^{h_{2}}$ and $\kappa_{\gamma \gamma}^{h_{2}}$ on the left panel and the correlation between $\kappa_{\gamma \gamma}^{h_{2}}$ and $\kappa_{\gamma Z}^{h_{2}}$ on the right panel, the SM value is indicated as a black box. $\kappa_{g g}, \kappa_{\gamma \gamma}$ and $\kappa_{\gamma Z}$ are the scaling factors for loop-induced channels which are defined by:

$\kappa_{j}^{2}=\Gamma^{j} / \Gamma_{S M}^{j}, \quad j=g g, \gamma \gamma, Z \gamma$ $j$ stands for a given loop process decay and $\Gamma^{j}$ is the partial decay rate. Note that $\kappa_{g g} \equiv \kappa_{g g}^{h_{2}}, \kappa_{\gamma \gamma} \equiv \kappa_{\gamma \gamma}^{h_{2}}, \kappa_{Z \gamma} \equiv \kappa_{Z \gamma}^{h_{2}}$ One can see that the deviations of $\kappa_{g g}^{h_{2}}, \kappa_{\gamma \gamma}^{h_{2}}$ and $\kappa_{\gamma Z}^{h_{2}}$ from the SM value can reach $15 \%$. Note that in both $\kappa_{\gamma \gamma}^{h_{2}}$ and $\kappa_{\gamma Z}^{h_{2}}$, we have, in most cases, a suppression of the rate compared to the value predicted by the SM. The figure also shows that we have suppression of $\kappa_{g g}^{h_{2}}, \kappa_{\gamma \gamma}^{h_{2}}$ and $\kappa_{\gamma Z}^{h_{2}}$ rate for $h_{2}$ with relatively large singlet component. We also stress that most of the cases $\kappa_{\gamma Z}^{h_{2}}<\kappa_{\gamma \gamma}^{h_{2}}$.

As we have seen in Fig. 5, decays of $h_{2}$ into SM particles such as $W W, Z Z, b \bar{b}$ and $\tau^{+} \tau^{-}$are consistent with LHC measurements with deviations from SM predictions that could goes up to $10-15 \%$. However, these deviations are mainly due to experimental uncertainties on all the LHC measurements which could be larger than $10 \%$ in some channels. Therefore, taking into account these uncertainties, there is still a room for the non-detected SM Higgs decays such as $h_{2} \rightarrow h_{1} h_{1}, A A, A A^{*}, A Z^{*}, H^{ \pm} W^{*}$. In our scan we assume that $m_{A}>62.5 \mathrm{GeV}$, therefore $h_{2} \rightarrow A A$ will not be open and $h_{2} \rightarrow A A^{*}$ is rather suppressed. We are only left with $h_{2} \rightarrow h_{1} h_{1}, A Z^{*}, H^{ \pm} W^{*}$ channels. As explained above, all these additional decays of the SM Higgs should not exceed $24 \%$.

We show in Fig. $7 \mathrm{Br}\left(h_{2} \rightarrow h_{1} h_{1}\right)$ as a function of $\operatorname{Br}\left(h_{2} \rightarrow Z^{*} A\right)+\operatorname{Br}\left(h_{2} \rightarrow W^{*} H^{ \pm}\right)$with $\kappa_{h_{2} h_{1} h_{1}}$ on the horizontal axis (left panel). While on the right panel we illustrate the singlet component of $h_{1}$ on the horizontal axis. Note that the couplings $h_{1} A Z$ and $h_{1} W^{\mp} H^{ \pm}$are exactly 

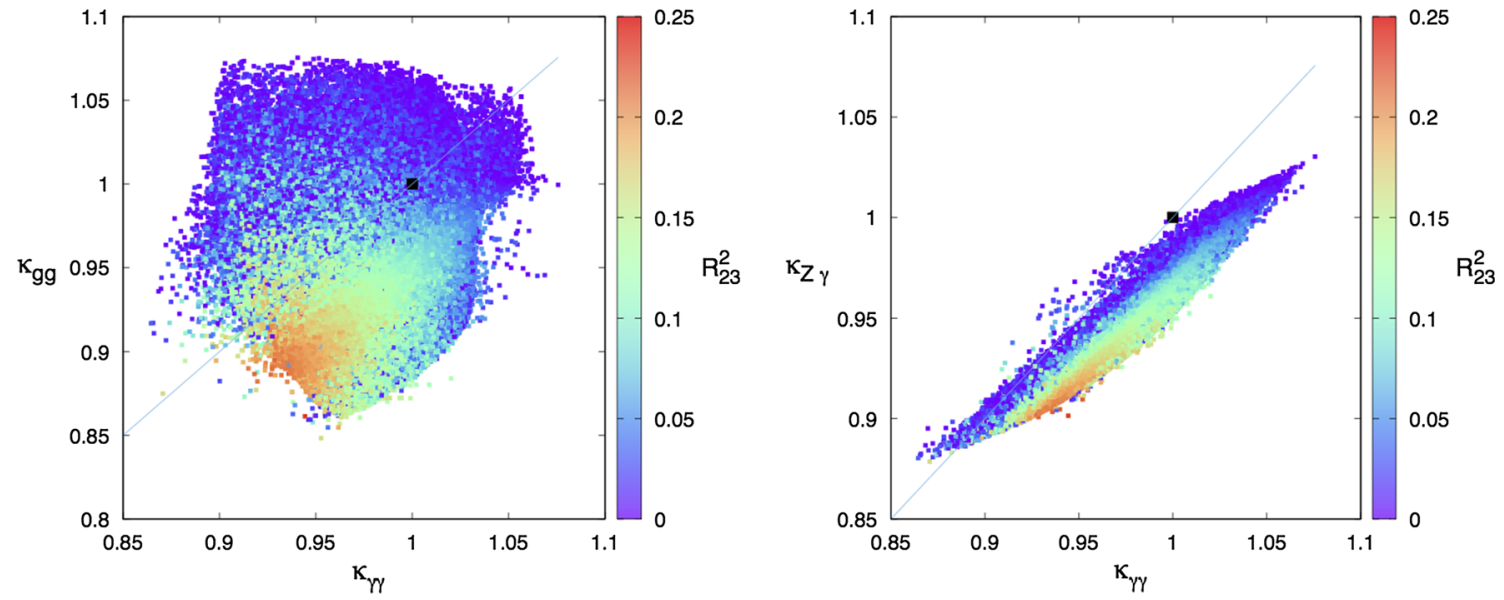

Fig. 6 Correlations: between $\kappa_{g g}^{h_{2}}$ and $\kappa_{\gamma \gamma}^{h_{2}}$ versus $R_{23}^{2}$ and between $\kappa_{\gamma \gamma}^{h_{2}}$ and $\kappa_{Z \gamma}^{h_{2}}$ as a function of the singlet component $R_{23}^{2}$ in N2HDM type-I at $95 \%$ C.L
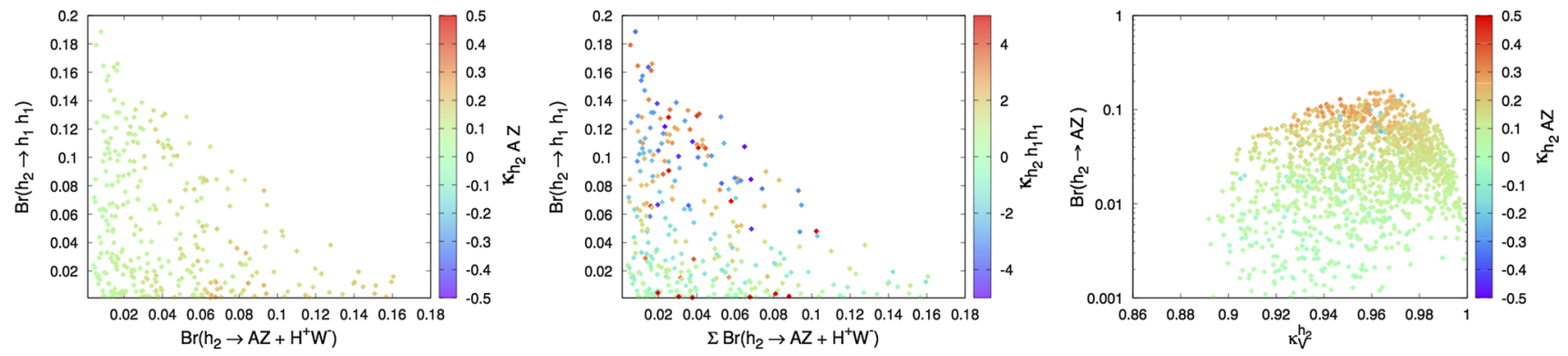

Fig. $7 \mathrm{Br}\left(h_{2} \rightarrow h_{1} h_{1}\right)$ and $\operatorname{Br}\left(h_{2} \rightarrow A Z+H^{+} W^{-}\right)$versus $\kappa_{h_{2} A Z}($ left $)$ and $\kappa_{h_{2}} h_{1} h_{1}$ (middle). On the right panel $B r\left(h_{2} \rightarrow A Z\right)$ versus $\kappa_{V}^{h_{2}}$ as a function of $\kappa_{h_{2}} A Z$ at $95 \%$ C.L in N2HDM type-I

the same (see Eq. (30)). Therefore, if $m_{A} \approx m_{H \pm}$ then $\operatorname{Br}\left(h_{2} \rightarrow Z^{*} A\right)$ and $\operatorname{Br}\left(h_{2} \rightarrow W^{*} H^{ \pm}\right)$are of the same order. The total amount for $\operatorname{Br}\left(h_{2} \rightarrow h_{1} h_{1}\right)+\operatorname{Br}\left(h_{2} \rightarrow\right.$ $\left.Z^{*} A\right)+B r\left(h_{2} \rightarrow W^{*} H^{ \pm}\right)$should not exceed $24 \%$ as requested from the non-detected decay of the SM Higgs, and this is rather clear from Fig. 7. The plots also display the correlation between $\operatorname{Br}\left(h_{2} \rightarrow h_{1} h_{1}\right)$ and $\operatorname{Br}\left(h_{2} \rightarrow\right.$ $\left.Z^{*} A\right)+\operatorname{Br}\left(h_{2} \rightarrow W^{* \mp} H^{ \pm}\right)$. When $\operatorname{Br}\left(h_{2} \rightarrow h_{1} h_{1}\right)$ is maximized, $\operatorname{Br}\left(h_{2} \rightarrow Z^{*} A\right)+\operatorname{Br}\left(h_{2} \rightarrow W^{* \mp} H^{ \pm}\right)$is minimal and vice verse. One can have also a configuration where both $\operatorname{Br}\left(h_{2} \rightarrow h_{1} h_{1}\right)$ and $\operatorname{Br}\left(h_{2} \rightarrow Z^{*} A\right)+\operatorname{Br}\left(h_{2} \rightarrow W^{* \mp} H^{ \pm}\right)$ are of the same size. In the case where both $\mathrm{A}$ and $H^{ \pm}$are heavier than $125 \mathrm{GeV}$, only $h_{2} \rightarrow h_{1} h_{1}$ would contribute to the non-detected decay of $h_{2}$.

On the middle panel of Fig. 7 it is clear that when the reduced coupling of $h_{2} h_{1} h_{1}$ is large, the branching ratio $\operatorname{Br}\left(h_{2} \rightarrow h_{1} h_{1}\right)$ is substantial which would provide an important production channel for $h_{1}$ from $h_{2}$ decay: $g g \rightarrow$ $h_{2} \rightarrow h_{1} h_{1}$ which could compete with the other production channels such as $p p \rightarrow W h_{1}$ and/or $p p \rightarrow\left\{h_{1} A, h_{1} H^{ \pm}\right\}$.

On the right panel of Fig. 7 we illustrate the correlation between $\operatorname{Br}\left(h_{2} \rightarrow Z^{*} A\right)$ and $\kappa_{V}^{h_{2}}$ as a function of $\kappa_{h_{2} A Z \text {. }}$ As one can see from the plot, and according to the sum rule
Eq. (30), when $h_{2} V V$ is full strength, then $\operatorname{Br}\left(h_{2} \rightarrow Z^{*} A\right)$ is suppressed.

We have seen previously that $\operatorname{Br}\left(h_{2} \rightarrow h_{1} h_{1}\right)$ could be significant and can reach $20 \%$ in some case. In the case where $h_{1}$ is dominated by the singlet component, it is well known that it is hard to produce it through the conventional channels such as ggF, VBF ect. Therefore, the process $g g \rightarrow h_{2}$ followed by the decay $h_{2} \rightarrow h_{1} h_{1}$ could be an important process for the production of $h_{1}$. In the case where $h_{1}$ is dominated by the singlet component, its decay to SM particle would be suppressed. In such case, it may be possible that $h_{1}$ would decay to a pair of photons which could proceed through charged Higgs loops. Therefore, the process $g g \rightarrow h_{2} \rightarrow h_{1} h_{1}$ could lead to a spectacular 4 photons final states. In Fig. 8 (left) we illustrate the branching fraction $\operatorname{Br}\left(h_{2} \rightarrow h_{1} h_{1}\right) \times \operatorname{Br}\left(h_{1} \rightarrow \gamma \gamma\right)^{2}$ as a function of $m_{h_{1}}$. As can be seen, such branching fraction could reach $10 \%$ in some cases. On the right panel of Fig. 8, we show the production cross section for $\sigma\left(g g \rightarrow h_{2}\right) \times B r\left(h_{2} \rightarrow\right.$ $\left.h_{1} h_{1}\right) \times \operatorname{Br}\left(h_{1} \rightarrow \gamma \gamma\right)^{2}$.

We note that for very small singlet component $R_{13} \approx 0$ where $h_{1}$ is fully dominated by the doublet components, one 

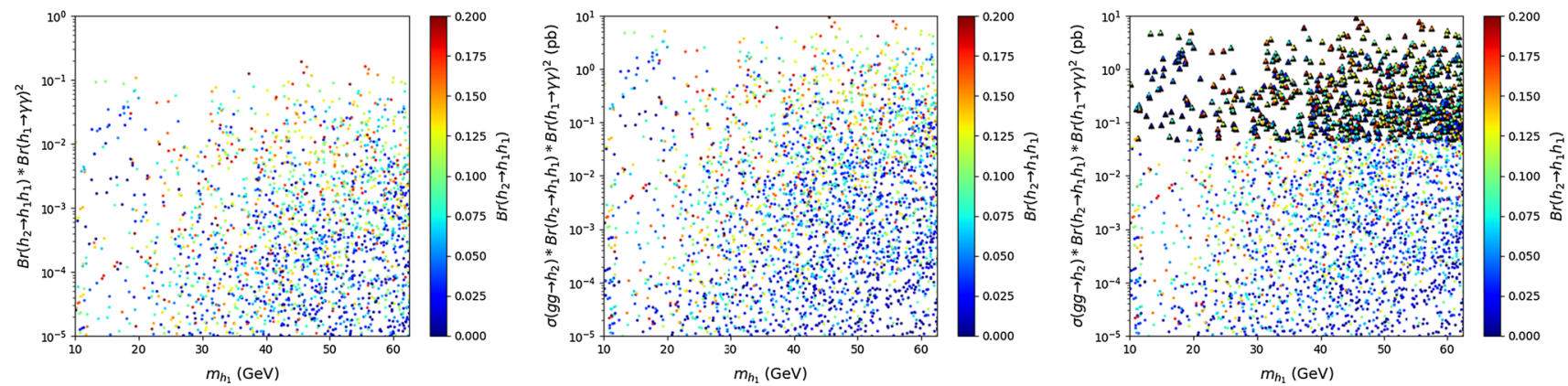

Fig. $8 \operatorname{Br}\left(h_{2} \rightarrow h_{1} h_{1}\right) \times \operatorname{Br}\left(h_{1} \rightarrow \gamma \gamma\right)^{2}$ (left panel) and $\sigma\left(g g \rightarrow h_{2}\right) \times \operatorname{Br}\left(h_{2} \rightarrow h_{1} h_{1}\right) \times \operatorname{Br}\left(h_{1} \rightarrow \gamma \gamma\right)^{2}$ (middle and right panel) as a function of $m_{h_{1}}$ versus $\operatorname{Br}\left(h_{2} \rightarrow h_{1} h_{1}\right)$ at $95 \%$ C.L in N2HDM type-I. Black triangles denote the excluded points from Fig. 9

could have sizeable $\operatorname{Br}\left(h_{2} \rightarrow h_{1} h_{1}\right)$ as it has been noticed in the usual 2HDM $[64,65]$.

Recently, ATLAS published their results for the search of new phenomena in events with at least three photons [32] based on $8 \mathrm{TeV}$ CM energy with $20.3 \mathrm{fb}^{-1}$. This search was used to put constraint on an N-MSSM scenario which leads to four photons final states $g g \rightarrow H \rightarrow a_{1} a_{1} \rightarrow 4 \gamma$ where a light pseudo-scalar, if dominated by singlet component, can decay fully into two photons with $100 \%$ branching ratio. Following this work, it has been demonstrated in [65] that the kinematic distributions for $q q \rightarrow H \rightarrow a_{1} a_{1} \rightarrow 4 \gamma$ and $q q \rightarrow H \rightarrow h_{1} h_{1} \rightarrow 4 \gamma$ with $h_{1}$ being CP-even are identical. Reference [65] also provide a projection for $14 \mathrm{TeV}$ $\mathrm{CM}$ energy. Therefore results from [32] can be applied to our four photons final states. In Fig. 9, we present our predictions for $p p \rightarrow h_{2} \rightarrow h_{1} h_{1} \rightarrow 4 \gamma$ for both $8 \mathrm{TeV}$ and $14 \mathrm{TeV}$ together with the $8 \mathrm{TeV}$ exclusion from ATLAS analysis. ATLAS projection for $14 \mathrm{TeV}$ is also shown in the lower band. It is clear that some benchmark points are already excluded by the $8 \mathrm{TeV}$ data and the $14 \mathrm{TeV}$ projection. However, several benchmarks are still alive.

$h_{3}$ decays

We now discuss $h_{3}$ decays. We show in Fig. 10 the branching fractions for $h_{3} \rightarrow f f, f=b, \tau, t$ and $h_{3} \rightarrow V V, V=$ $\gamma, Z, W$ as a function of singlet component $R_{33}$ and $m_{h_{3}}$. It is clear that $h_{3}$ is dominated by singlet component. One can see that before reaching the $t \bar{t}$ threshold, $h_{3} \rightarrow W W$ could be the dominant decay mode of $h_{3}$ with a branching which can reach up to $80 \%$, while $h_{3} \rightarrow Z Z$ goes up to $20 \%$ and in such cases $h_{3} \rightarrow h_{1} h_{1}$ is suppressed. After reaching $t \bar{t}$ threshold, $h_{3} \rightarrow t \bar{t}$ can be slightly larger than $10 \%$ for large $m_{h_{3}}$ mass.

We now discuss Higgs to Higgs decays, such as $h_{3} \rightarrow$ $h_{1} h_{1}, h_{1} h_{2}, h_{2} h_{2}$ and $h_{3} \rightarrow Z A, W^{ \pm} H^{\mp}$. In Fig. 11 (upper plot) we illustrate the branching ratio of $h_{3} \rightarrow h_{1} h_{1}$ (left), $h_{3} \rightarrow h_{2} h_{2}$ (middle) and their correlation (right). From the left panel, one can see that $\operatorname{Br}\left(h_{3} \rightarrow h_{1} h_{1}\right)$ can be substan-

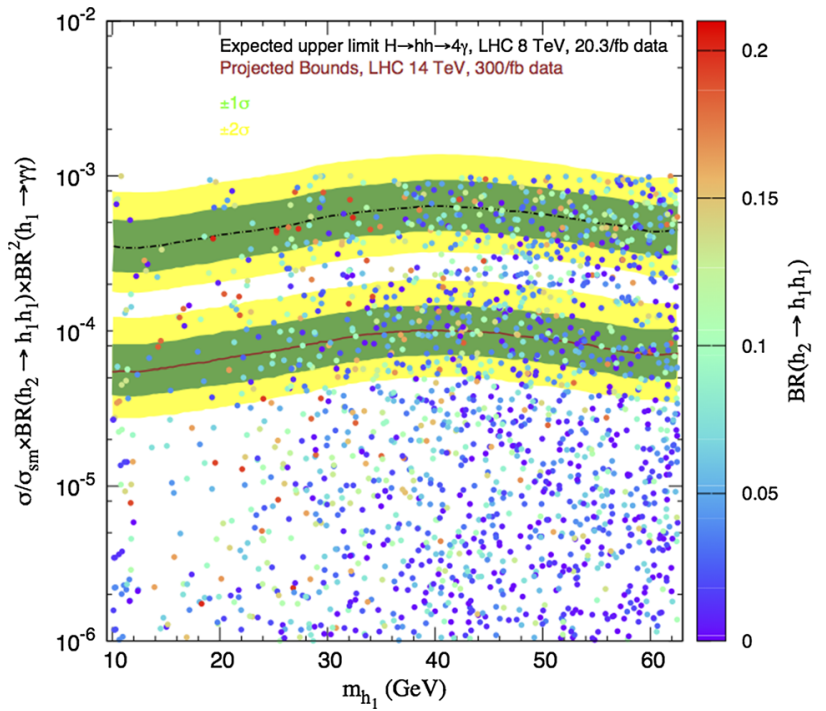

Fig. 9 Upper limit at 95\% CL on $\frac{\sigma\left(h_{2}\right)}{\sigma_{s m}} \times \operatorname{Br}\left(h_{2} \rightarrow h_{1} h_{1} \rightarrow 4 \gamma\right)$ as a function of $m_{h_{1}}$ vs. $\operatorname{Br}\left(h_{2} \rightarrow h_{1} h_{1}\right)$ from ATLAS searches at $8 \mathrm{TeV}$ (upper band) and the projection for $14 \mathrm{TeV}$ (lower band) taken from [65]. The green and yellow color indicate the allowed regions at $68 \%$ and $95 \%$, respectively

tial and becomes the dominant decay mode, while from the middle panel it is clear that $\operatorname{Br}\left(h_{3} \rightarrow h_{2} h_{2}\right)$ can reach $30 \%$ as a maximal value. In the case where $\operatorname{Br}\left(h_{3} \rightarrow h_{1} h_{1}\right)$ is the dominant decay, then one can have a new production mechanism for $h_{1}$, namely: $p p \rightarrow h_{3} \rightarrow h_{1} h_{1}$. This production channel might be useful for the case where $h_{1}$ has large singlet component in which case it will be challenging to produce it in the conventional channels.

In the lower panels of Fig. 11 we display the correlation between $\operatorname{Br}\left(h_{3} \rightarrow h_{1} h_{1}\right), \operatorname{Br}\left(h_{3} \rightarrow h_{2} h_{2}\right), \operatorname{Br}\left(h_{3} \rightarrow A A\right)$, $\operatorname{Br}\left(h_{3} \rightarrow h_{1} h_{2}\right)$ as well as with $\operatorname{Br}\left(h_{3} \rightarrow W W\right)$.

It is clear that one can have a scenario where both $\operatorname{Br}\left(h_{3} \rightarrow h_{2} h_{2}\right)$ and $\operatorname{Br}\left(h_{3} \rightarrow A A\right)$ are rather large with branching fractions of the order $40 \%$. It is also clear that when $\operatorname{Br}\left(h_{3} \rightarrow h_{1} h_{1}\right)$ and $\operatorname{Br}\left(h_{3} \rightarrow h_{2} h_{2}\right)$ are suppressed then $\operatorname{Br}\left(h_{3} \rightarrow W W, Z Z\right)$ would become slightly large. 

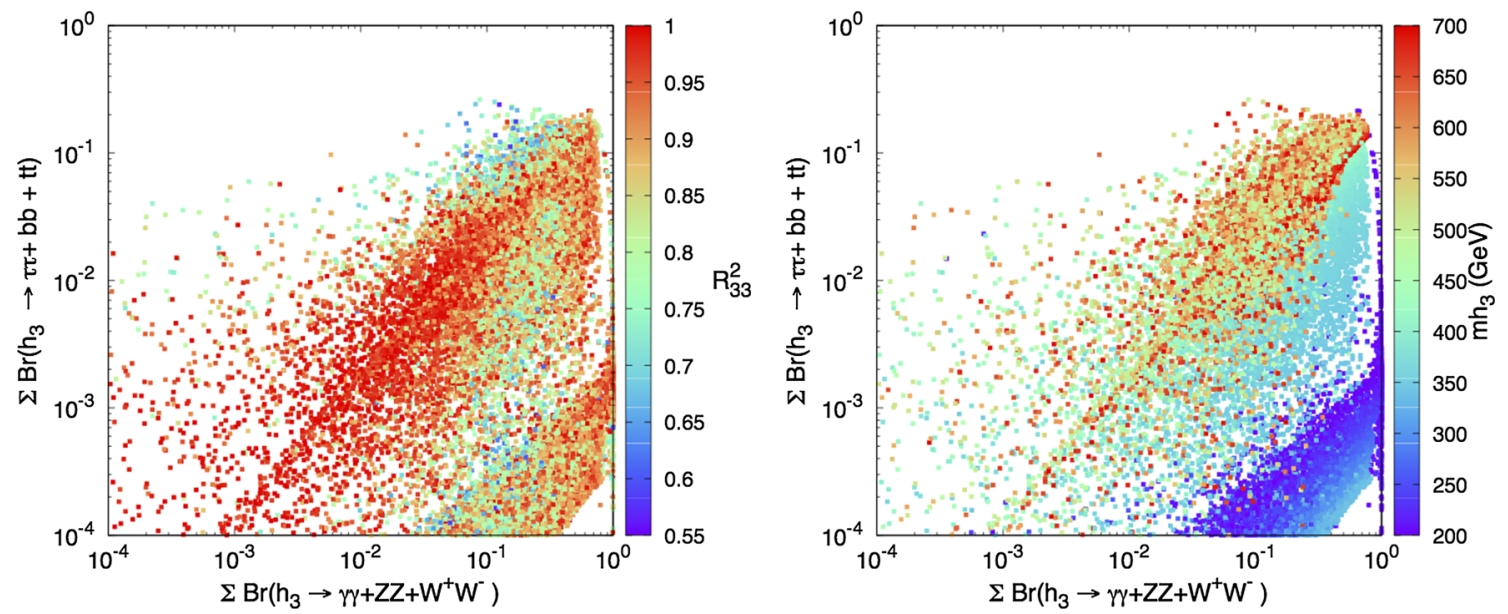

Fig. 10 Correlation between $\operatorname{Br}\left(h_{3} \rightarrow f f\right)$ and $\operatorname{Br}\left(h_{3} \rightarrow V V\right)$ versus $R_{33}^{2}$ (left) and $m_{h_{3}}$ (right) (GeV) in N2HDM type-I
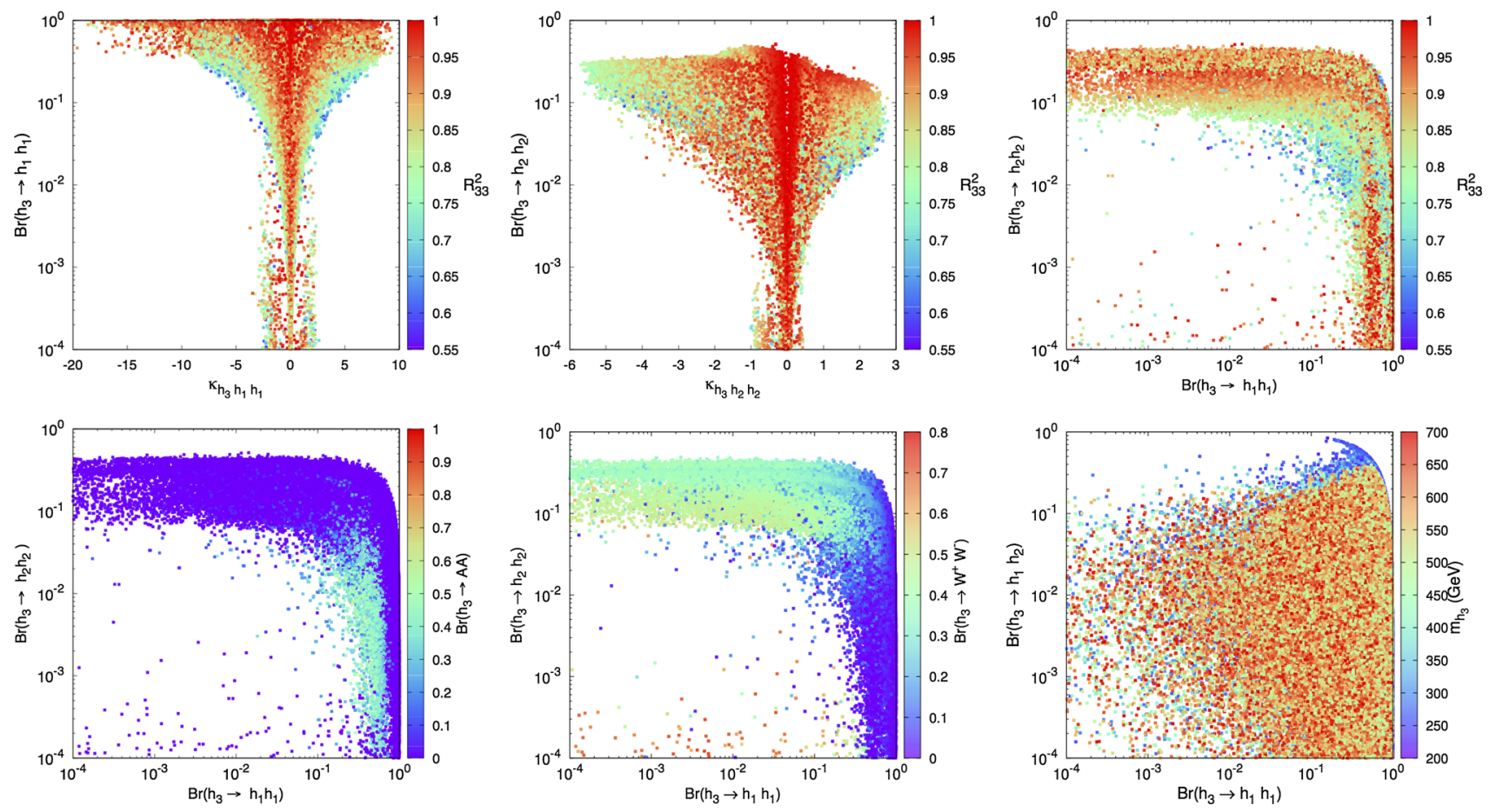

Fig. 11 Upper panels: (left) $\operatorname{Br}\left(h_{3} \rightarrow h_{1} h_{1}\right)$ as a function of $\kappa_{h_{3}} h_{1} h_{1}$ and (middle) $\operatorname{Br}\left(h_{3} \rightarrow h_{2} h_{2}\right)$ as a function of $\kappa_{h_{3} h_{2} h_{2}}$ with $R_{33}^{2}$ displayed on the vertical and (right) the correlation between $\operatorname{Br}\left(h_{3} \rightarrow\right.$ $\left.h_{1} h_{1}\right)$ and $\operatorname{Br}\left(h_{3} \rightarrow h_{2} h_{2}\right)$. Lower panels: correlation between

$\operatorname{Br}\left(h_{3} \rightarrow h_{1} h_{1}\right)$ and $\operatorname{Br}\left(h_{3} \rightarrow h_{2} h_{2}\right)$ as a function of $\operatorname{Br}\left(h_{3} \rightarrow A A\right)$ (lower-left), as a function of $B r\left(h_{3} \rightarrow W W\right.$ ) (lower-middle) and correlation between $\operatorname{Br}\left(h_{3} \rightarrow h_{1} h_{1}\right)$ and $\operatorname{Br}\left(h_{3} \rightarrow h_{1} h_{2}\right)$ as a function of $m_{h_{3}}$

In Fig. 12 we show the branching fractions of $h_{3} \rightarrow A Z$ and $h_{3} \rightarrow H^{ \pm} W^{\mp}$ versus $R_{33}^{2}$. As one can see from the plots and according to the sum-rule Eq. (30), when $h_{3}$ is dominated by the singlet component; $R_{33} \approx 1$, then both $h_{3} Z A$ and $h_{3} W^{\mp} H^{ \pm}$couplings are suppressed resulting in a small branching ratio for both channels. For $R_{33}$ away from 1 , the branching fraction $h_{3} \rightarrow A Z$ and $h_{3} \rightarrow H^{ \pm} W^{\mp}$ can be in the range of $10-40 \%$ in some cases.
Given that $\operatorname{Br}\left(h_{3} \rightarrow h_{1} h_{1}\right)$ can be sizeable, one can look to the amount of production cross section that one can get from $h_{3}$ production followed by $h_{3}$ decay into a pair of $h_{1}$. We illustrate in Fig. 13 both $p p \rightarrow h_{3} \rightarrow h_{2} h_{2}$ (left), $p p \rightarrow$ $h_{3} \rightarrow h_{1} h_{1}$ (middle) and $p p \rightarrow h_{3} \rightarrow h_{1} h_{2}$ (right) where the production cross section of $h_{3}$ is computed at $\sqrt{s}=$ $13 \mathrm{TeV}$ with Sushi $[66,67]$.

One can see that the production rate of $h_{1}$ is large especially in the mass range $200 \mathrm{GeV} \lesssim m_{h_{3}} \lesssim 250 \mathrm{GeV}$ when 

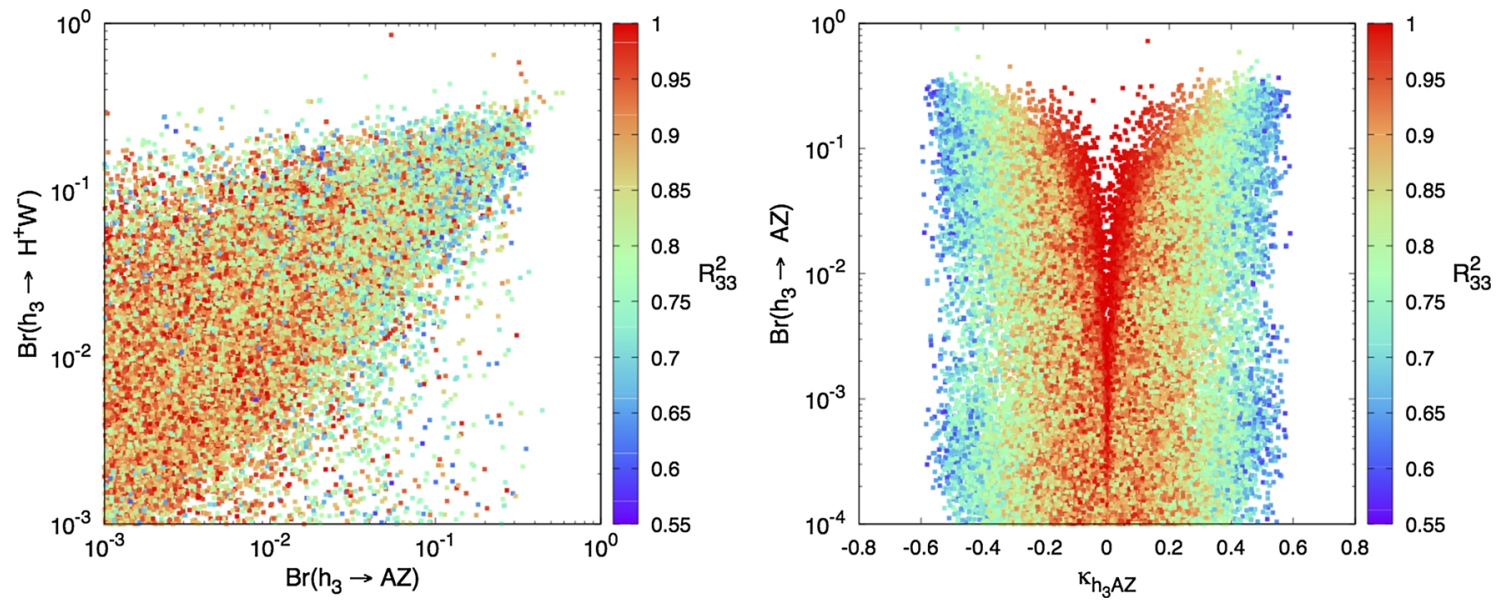

Fig. 12 Correlation between $\operatorname{Br}\left(h_{3} \rightarrow A Z\right)$ and $\operatorname{Br}\left(h_{3} \rightarrow H^{ \pm} W^{\mp}\right)$ versus $R_{33}^{2}$ (left panel) and $B r\left(h_{3} \rightarrow A Z\right)$ as a function of $\kappa_{h_{3} A Z}$ and $R_{33}^{2}$ (right panel)
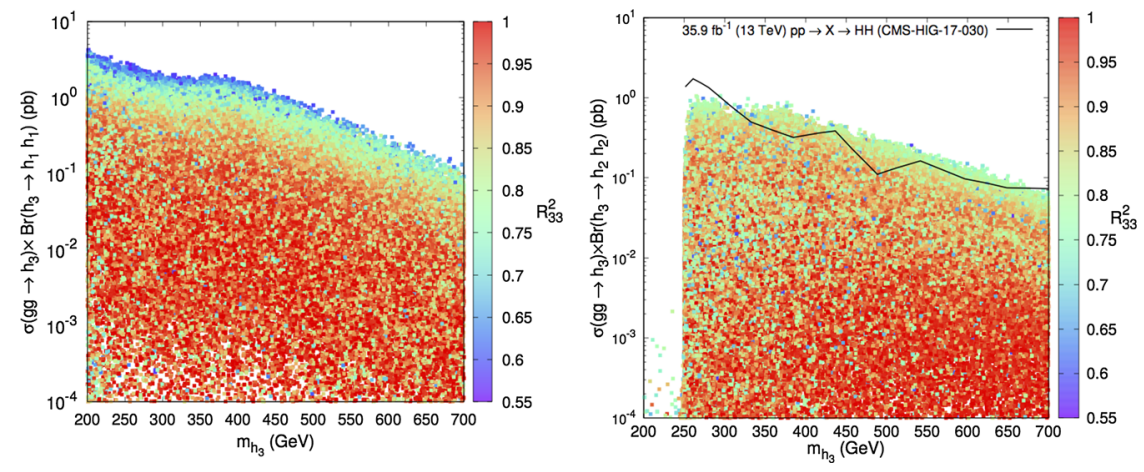

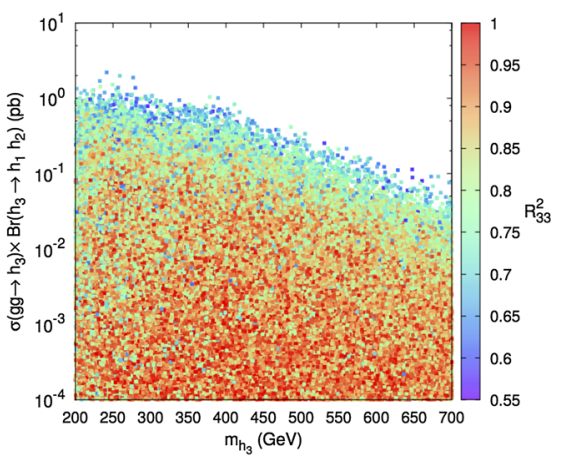

Fig. $13 \sigma\left(p p \rightarrow h_{3}\right) \times \operatorname{Br}\left(h_{3} \rightarrow h_{2} h_{2}\right)\left(\right.$ left panel), $\sigma\left(p p \rightarrow h_{3}\right) \times$ $\operatorname{Br}\left(h_{3} \rightarrow h_{1} h_{1}\right)$ (middle panel) and $\sigma\left(p p \rightarrow h_{3}\right) \times \operatorname{Br}\left(h_{3} \rightarrow h_{1} h_{2}\right)$ (right panel) as a function of $m_{h_{3}}(\mathrm{GeV})$ and $R_{33}^{2}$ in N2HDM type-I.

the decay channel $h_{3} \rightarrow h_{1} h_{1}$ is kinematically allowed and both $h_{3} \rightarrow h_{2} h_{2}$ and $h_{3} \rightarrow t \bar{t}$ are closed. The same behavior is observed in the middle panel, where the magnitude in the cross section is larger in the mass range $250 \mathrm{GeV} \lesssim m_{h_{3}} \lesssim$ $350 \mathrm{GeV}$ when the decay channel $h_{3} \rightarrow h_{2} h_{2}$ is opened and $h_{3} \rightarrow t \bar{t}$ mode is closed. However, one can see that after applying the constraints on the heavy scalar resonances decaying into two SM-like scalars with a mass of $\sim 125$ $\mathrm{GeV}$ [68], some of the parameter space points are already excluded.

\section{Conclusions}

We have studied the two Higgs doublet model extended with a real singlet scalar. The spectrum contains $3 \mathrm{CP}$-even $h_{1,2,3}$, one CP-odd and a pair of charged Higgs. We derive full set of perturbative unitarity constraints, boundedness from below constraints as well as the oblique parameters $\mathrm{S}, \mathrm{T}$ and $\mathrm{U}$.
Solid line is the observed $95 \%$ CL exclusion limits on the production of a narrow width spin zero resonance decaying into a pair of Higgs bosons [68]

In our analysis, we concentrate on the case where $h_{2}$ is the SM Higgs boson observed at the LHC and assume that $h_{1}$ is lighter than $125 \mathrm{GeV}$. We study the consistency of our scenario with both LHC data taken at $8 \mathrm{TeV}$ and $13 \mathrm{TeV}$ as well as with all the available LEP-II and Tevatron data. We have shown in the framework of N2HDM that:

- $h_{1}$ can be quasi-fermiophobic and would decay dominantly into two photons.

- LHC data still allow a room for the non-detected decays of the SM-Higgs $h_{2} \rightarrow h_{1} h_{1}$ and others with a branching ratio of the order which can reach $24 \%$. Such decay followed by two photons decay of $h_{1}$ could lead to four photons signature, namely $p p \rightarrow h_{2} \rightarrow h_{1} h_{1} \rightarrow 4 \gamma$.

- Comparison of ATLAS data with our four photons signal show that there is a large area of parameter space that still escapes ATLAS data

We have also shown that in N2HDM type I, $h_{2}$ and $h_{3}$ can decay to some exotic modes such as $h_{2,3} \rightarrow h_{1} h_{1}$, 
$h_{2,3} \rightarrow Z A$ and $h_{2,3} \rightarrow W \pm H^{\mp}$ with substantial branching ratio. The production process $g g \rightarrow h_{2,3}$ followed by the decays $h_{2,3} \rightarrow h_{1} h_{1}, h_{1} h_{2}$ could be sizeable and could be an important source of production of $h_{1}$ in the case where $h_{1}$ have a large singlet component where it is rather difficult to produce it using the conventional channel.

Acknowledgements This work is supported by the Moroccan Ministry of Higher Education and Scientific Research MESRSFC and CNRST: Projet PPR/2015/6.

Data Availability Statement This manuscript has no associated data or the data will not be deposited. [Authors' comment: This is a theoretical study that has no associated data.]

Open Access This article is licensed under a Creative Commons Attribution 4.0 International License, which permits use, sharing, adaptation, distribution and reproduction in any medium or format, as long as you give appropriate credit to the original author(s) and the source, provide a link to the Creative Commons licence, and indicate if changes were made. The images or other third party material in this article are included in the article's Creative Commons licence, unless indicated otherwise in a credit line to the material. If material is not included in the article's Creative Commons licence and your intended use is not permitted by statutory regulation or exceeds the permitted use, you will need to obtain permission directly from the copyright holder. To view a copy of this licence, visit http://creativecomm ons.org/licenses/by/4.0/.

Funded by SCOAP ${ }^{3}$.

\section{Appendix A: BFB constraints}

Consider for instance the following case in which there is no coupling between doublets $H_{i}$ and singlet $S$ Higgs bosons, i.e. $\lambda_{3}=\lambda_{4}=\lambda_{5}=\lambda_{7}=\lambda_{8}=0$, it is obvious that

$\lambda_{1}>0 \& \lambda_{2}>0 \& \lambda_{6}>0$

To proof the necessary and sufficient BFB conditions, we adopt a different parameterization of the fields that will turn out to be particularly convenient to entirely solve the problem. For that we define:

$$
\begin{aligned}
r & \equiv \sqrt{H_{1}^{\dagger} H_{1}+H_{2}^{\dagger} H_{2}+S^{\dagger} S} \\
H_{1}^{\dagger} H_{1} & \equiv r^{2} \cos ^{2} \theta \sin ^{2} \phi \\
H_{2}^{\dagger} H_{2} & \equiv r^{2} \sin ^{2} \theta \sin ^{2} \phi \\
S^{\dagger} S & \equiv r^{2} \cos ^{2} \phi . \\
\frac{H_{1}^{\dagger} H_{2}}{\left|H_{1}\right|\left|H_{2}\right|} & \equiv \xi e^{i \psi} .
\end{aligned}
$$

Obviously, when $H_{1}, H_{2}$ and $S$ scan all the field space, the radius $r$ scans the domain $[0, \infty[$, the angle $\theta \in[0,2 \pi]$ and the angle $\phi \in\left[0, \frac{\pi}{2}\right]$. Moreover, one can show that $\frac{H_{1}^{\dagger}}{\left|H_{1}\right|} \cdot \frac{H_{2}}{\left|H_{2}\right|}$ is a product of unit spinor, so that $\xi \in[0,1]$.
With this parameterization, one can cast $V^{(4)}\left(H_{1}, H_{2}, S\right)$ in the following simple form,

$$
\begin{aligned}
V^{(4)} & \left(r, c_{\theta}^{2}, s_{\phi}^{2}, c_{2 \psi}, \xi\right) \\
= & r^{4}\left\{\frac{\lambda_{1}}{2} c_{\theta}^{4} s_{\phi}^{4}+\frac{\lambda_{2}}{2} s_{\theta}^{4} s_{\phi}^{4}+\lambda_{3} c_{\theta}^{2} s_{\theta}^{2} s_{\phi}^{4}+\lambda_{4} c_{\theta}^{2} s_{\theta}^{2} s_{\phi}^{4} \xi^{2}\right. \\
& +\frac{\lambda_{5}}{2} c_{\theta}^{2} s_{\theta}^{2} s_{\phi}^{4} \xi^{2}\left(e^{2 i \psi}+e^{-2 i \psi}\right)+\frac{\lambda_{6}}{8} c_{\phi}^{4} \\
& \left.+\frac{1}{2}\left(\lambda_{7} c_{\theta}^{2} s_{\phi}^{2} c_{\phi}^{2}+\lambda_{8} s_{\theta}^{2} s_{\phi}^{2} c_{\phi}^{2}\right)\right\}
\end{aligned}
$$

Let define again:

$x \equiv \cos ^{2} \theta, \quad y \equiv \sin ^{2} \phi, \quad z \equiv \cos 2 \psi$

which allows to write

$$
\begin{aligned}
V^{(4)} & (x, y, z, \xi) \\
= & \left\{\frac{\lambda_{1}}{2} x^{2}+\frac{\lambda_{2}}{2}(1-x)^{2}+\lambda_{3} x(1-x)\right. \\
& \left.+\lambda_{4} x(1-x) \xi^{2}+\lambda_{5} x(1-x) \xi^{2} z\right\} y^{2} \\
& +\frac{\lambda_{6}}{8}(1-y)^{2} \\
& +\left\{\frac{\lambda_{7}}{2} x+\frac{\lambda_{8}}{2}(1-x)\right\} y(1-y)
\end{aligned}
$$

it is easy to find the constraint conditions by studying $V^{(4)}(x, y, z, \xi)$ as a quadratic function using the fact that:

$$
\begin{aligned}
& f(\zeta)=a \zeta^{2}+b(1-\zeta)^{2}+c \zeta(1-\zeta), \quad \zeta \in(0,1) \\
& \quad \Leftrightarrow \quad a>0, b>0 \text { and } c+2 \sqrt{a b}>0
\end{aligned}
$$

we can deduce the set of constraints as:

$$
\begin{aligned}
A \equiv & \frac{\lambda_{1}}{2} x^{2}+\frac{\lambda_{2}}{2}(1-x)^{2}+\lambda_{3} x(1-x)+ \\
& \lambda_{4} x(1-x) \xi^{2}+\lambda_{5} x(1-x) \xi^{2} z>0 \\
B \equiv & \frac{\lambda_{6}}{8}>0 \\
C \equiv & \frac{\lambda_{7}}{2} x+\frac{\lambda_{8}}{2}(1-x)>-2 \sqrt{A B}
\end{aligned}
$$

the simple condition can be extracted from Eq. (A12), which imply $\lambda_{6}>0$. For $A>0$ one can use Eq. (A10) again to get the ordinary $2 \mathrm{HDM}$ BFB constraints taking into account if $\xi=0 ; 1$ and $z=-1 ; 1$ :

$$
\begin{aligned}
\lambda_{1}, \lambda_{2} & >0 \\
\lambda_{3}+\sqrt{\lambda_{1} \lambda_{2}} & >0 \\
\lambda_{3}+\lambda_{4}+\left|\lambda_{5}\right|+\sqrt{\lambda_{1} \lambda_{2}} & >0
\end{aligned}
$$

For the Eq. (A13), we can consider two scenarios:

$-\operatorname{scenario}(1): \lambda_{7}$ and $\lambda_{8}>0$ starting with the fact that $x=\cos ^{2} \theta>0$ and $1-x=$ $\sin ^{2} \theta>0$, thus $C>0 \rightarrow \lambda_{7}, \lambda_{8}>0$ imply that $A B>$ 0 which already done in Eqs. (A11, A12). 
$-\operatorname{scenario}(2): \lambda_{7}$ or $\lambda_{8}<0$

this scenario implies $\lambda_{7}$ or $\backslash$ and $\lambda_{8} \leq 0$ and it leads to two cases:

$C>-2 \sqrt{A B} \Leftrightarrow\left\{\begin{array}{l}-2 \sqrt{A B}<C<2 \sqrt{A B} \quad \text { (i) } \\ C>2 \sqrt{A B}\end{array}\right.$

For scenario(i), we can rewrite it like this:

$$
\begin{aligned}
& \left(\lambda_{1} \lambda_{6}-\lambda_{7}^{2}\right) x^{2}+\left(\lambda_{2} \lambda_{6}-\lambda_{8}^{2}\right)(1-x)^{2} \\
& \quad+2\left\{\lambda_{6}\left(\lambda_{3}+\lambda_{4} \xi^{2}+\lambda_{5} \xi^{2} z\right)-\lambda_{7} \lambda_{8}\right\} x(1-x)>0
\end{aligned}
$$

by applying the lemme given by Eq. (A10), we get the following new constraints:

$$
\begin{aligned}
& \lambda_{7}^{2}<\lambda_{1} \lambda_{6} \\
& \lambda_{8}^{2}<\lambda_{2} \lambda_{6} \\
& \lambda_{6}\left(\lambda_{3}+\lambda_{4} \xi^{2}+\lambda_{5} \xi^{2} z\right)-\lambda_{7} \lambda_{8} \\
& \quad+\sqrt{\left(\lambda_{1} \lambda_{6}-\lambda_{7}^{2}\right)\left(\lambda_{2} \lambda_{6}-\lambda_{8}^{2}\right)}>0
\end{aligned}
$$

Both Eqs. (A19) and (A20) gives constraints on $\lambda_{7}$ and $\lambda_{8}$ :

$$
-\sqrt{\lambda_{1} \lambda_{6}}\left(-\sqrt{\lambda_{2} \lambda_{6}}\right)<\lambda_{7}\left(\lambda_{8}\right)<\sqrt{\lambda_{1} \lambda_{6}}\left(\sqrt{\lambda_{2} \lambda_{6}}\right)
$$

since $\xi=0 ; 1$ and $z=-1 ; 1$, the latest equations leads to

$$
\begin{aligned}
& \lambda_{3} \lambda_{6}-\lambda_{7} \lambda_{8}+\sqrt{\left(\lambda_{1} \lambda_{6}-\lambda_{7}^{2}\right)\left(\lambda_{2} \lambda_{6}-\lambda_{8}^{2}\right)}>0 \\
& \lambda_{6}\left(\lambda_{3}+\lambda_{4}+\left|\lambda_{5}\right|\right)-\lambda_{7} \lambda_{8} \\
& \quad+\sqrt{\left(\lambda_{1} \lambda_{6}-\lambda_{7}^{2}\right)\left(\lambda_{2} \lambda_{6}-\lambda_{8}^{2}\right)}>0
\end{aligned}
$$

In the other hand, scenario(ii) leads to a contradiction because it implies that $\lambda_{7}>0$ and $\lambda_{8}>0$, which is not our case.

\section{Appendix B: Unitarity constraints}

The first submatrix $\mathcal{M}_{1}$ corresponds to scattering whose initial and final states are one of the following : $\left(\phi_{1}^{+} \phi_{2}^{-}, \phi_{2}^{+} \phi_{1}^{-}\right.$, $\left.\phi_{1} \chi_{2}, \phi_{2} \chi_{1}, \phi_{s} \chi_{1}, \phi_{s} \chi_{2}, \phi_{1} \phi_{2}, \phi_{1} \phi_{s}, \phi_{2} \phi_{s}, \chi_{1} \chi_{2}\right)$. We have to write out the full matrix, one finds,

$$
\mathcal{M}_{1}\left(\begin{array}{ccccccccccc}
\lambda_{34}^{+} & 2 \lambda_{5} & -i \frac{\lambda_{45}^{-}}{2} & +i \frac{\lambda_{45}^{-}}{2} & 0 & 0 & \frac{\lambda_{45}^{+}}{2} & 0 & 0 & \frac{\lambda_{45}^{+}}{2} \\
2 \lambda_{5} & \lambda_{34}^{+} & +i \frac{\lambda_{45}^{-}}{2} & -i \frac{\lambda_{45}^{-}}{2} & 0 & 0 & \frac{\lambda_{45}^{+}}{2} & 0 & 0 & \frac{\lambda_{45}^{+}}{2} \\
+i \frac{\lambda_{45}^{-}}{2} & -i \frac{\lambda_{45}^{-}}{2} & \lambda_{L} & \lambda_{5} & 0 & 0 & 0 & 0 & 0 & 0 \\
-i \frac{\lambda_{45}^{-}}{2} & +i \frac{\lambda_{45}^{-}}{2} & \lambda_{5} & \lambda_{L} & 0 & 0 & 0 & 0 & 0 & 0 \\
0 & 0 & 0 & 0 & \frac{\lambda_{7}}{2} & 0 & 0 & 0 & 0 & 0 \\
0 & 0 & 0 & 0 & 0 & \frac{\lambda_{8}}{2} & 0 & 0 & 0 & 0 \\
\frac{\lambda_{45}^{+}}{2} & \frac{\lambda_{45}^{+}}{2} & 0 & 0 & 0 & 0 & \lambda & 0 & 0 & \lambda_{5} \\
0 & 0 & 0 & 0 & 0 & 0 & 0 & \frac{\lambda_{7}}{2} & 0 & 0 \\
0 & 0 & 0 & 0 & 0 & 0 & 0 & 0 & \frac{\lambda_{8}}{2} & 0 \\
\frac{\lambda_{45}^{+}}{2} & \frac{\lambda_{45}^{+}}{2} & 0 & 0 & 0 & 0 & \lambda_{5} & 0 & 0 & \lambda
\end{array}\right)
$$

where $\lambda=\lambda_{3}+\lambda_{4}+\lambda_{5}$ and $\lambda_{L}=\lambda_{3}+\lambda_{4}-\lambda_{5}$. We find that $\mathcal{M}_{1}$ has the following eigenvalues given by :

$a_{1}=\lambda_{3}+\lambda_{4}$

$a_{2}=\lambda_{3}-\lambda_{5}$

$a_{3}=\lambda_{3}+\lambda_{5}$

$a_{4}=\frac{\lambda_{7}}{2}$

$a_{5}=\frac{\lambda_{8}}{2}$

$a_{ \pm}=\lambda_{3}+2 \lambda_{4} \pm 3 \lambda_{5}$

The second submatrix $\mathcal{M}_{2}$ corresponds to scattering with one of the following initial and final states: $\left(\phi_{1}^{+} \phi_{1}^{-}, \phi_{2}^{+} \phi_{2}^{-}\right.$, $\left.\frac{\phi_{1} \phi_{1}}{\sqrt{2}}, \frac{\phi_{2} \phi_{2}}{\sqrt{2}}, \frac{\phi_{s} \phi_{s}}{\sqrt{2}}, \frac{\chi_{1} \chi_{1}}{\sqrt{2}}, \frac{\chi_{2} \chi_{2}}{\sqrt{2}}\right)$, where the $\sqrt{2}$ accounts for identical particle statistics. One finds that $\mathcal{M}_{2}$ is given by:

$$
\mathcal{M}_{2}=\left(\begin{array}{ccccccc}
2 \lambda_{1} & \lambda_{34}^{+} & \frac{\lambda_{1}}{\sqrt{2}} & \frac{\lambda_{3}}{\sqrt{2}} & \frac{\lambda_{7}}{2 \sqrt{2}} & \frac{\lambda_{1}}{\sqrt{2}} & \frac{\lambda_{3}}{\sqrt{2}} \\
\lambda_{34}^{+} & 2 \lambda_{2} & \frac{\lambda_{3}}{\sqrt{2}} & \frac{\lambda_{2}}{\sqrt{2}} & \frac{\lambda_{8}}{2 \sqrt{2}} & \frac{\lambda_{3}}{\sqrt{2}} & \frac{\lambda_{2}}{\sqrt{2}} \\
\frac{\lambda_{1}}{\sqrt{2}} & \frac{\lambda_{3}}{\sqrt{2}} & \frac{3 \lambda_{1}}{2} & \frac{\lambda}{2} & \frac{\lambda_{7}}{4} & \frac{\lambda_{1}}{2} & \frac{\lambda_{L}}{2} \\
\frac{\lambda_{3}}{\sqrt{2}} & \frac{\lambda_{2}}{\sqrt{2}} & \frac{\lambda}{2} & \frac{3 \lambda_{2}}{2} & \frac{\lambda_{8}}{4} & \frac{\lambda_{L}}{2} & \frac{\lambda_{2}}{2} \\
\frac{\lambda_{7}}{2 \sqrt{2}} & \frac{\lambda_{8}}{2 \sqrt{2}} & \frac{\lambda_{7}}{4} & \frac{\lambda_{8}}{4} & \frac{3 \lambda_{6}}{8} & \frac{\lambda_{7}}{4} & \frac{\lambda_{8}}{4} \\
\frac{\lambda_{1}}{\sqrt{2}} & \frac{\lambda_{3}}{\sqrt{2}} & \frac{\lambda_{1}}{2} & \frac{\lambda_{L}}{2} & \frac{\lambda_{7}}{4} & \frac{3 \lambda_{1}}{2} & \frac{\lambda}{2} \\
\frac{\lambda_{3}}{\sqrt{2}} & \frac{\lambda_{2}}{\sqrt{2}} & \frac{\lambda_{L}}{2} & \frac{\lambda_{2}}{2} & \frac{\lambda_{8}}{4} & \frac{\lambda}{2} & \frac{3 \lambda_{2}}{2}
\end{array}\right)
$$


Despite its apparently complicated structure, three eigenvalues of $\mathcal{M}_{2}$ are located as roots of the following cubic polynomial equation:

$2 x^{3}+\left(-6 \lambda_{1}-6 \lambda_{2}-\lambda_{6}\right) x^{2}+\left(18 \lambda_{1} \lambda_{2}-8 \lambda_{3}^{2}-8 \lambda_{3} \lambda_{4}\right.$

$\left.-2 \lambda_{4}^{2}+3 \lambda_{1} \lambda_{6}+3 \lambda_{2} \lambda_{6}-\lambda_{7}^{2}-\lambda_{8}^{2}\right) x-9 \lambda_{1} \lambda_{2} \lambda_{6}+4 \lambda_{3}^{2} \lambda_{6}$

$+4 \lambda_{3} \lambda_{4} \lambda_{6}+\lambda_{4}^{2} \lambda_{6}+3 \lambda_{2} \lambda_{7}^{2}-4 \lambda_{3} \lambda_{7} \lambda_{8}$

$-2 \lambda_{4} \lambda_{7} \lambda_{8}+3 \lambda_{1} \lambda_{8}^{2}=0$

Those roots are denoted as $b_{1}, b_{2}$ and $b_{3}$. The remaining five eigenvalues of $\mathcal{M}_{2}$ are as follows:

$b_{4}=\frac{\lambda_{6}}{4}$

$b_{ \pm}=\frac{1}{2}\left(\lambda_{1}+\lambda_{2} \pm \sqrt{\left(\lambda_{1}-\lambda_{2}\right)^{2}+4 \lambda_{4}^{2}}\right)$

$f_{ \pm}=\frac{1}{2}\left(\lambda_{1}+\lambda_{2} \pm \sqrt{\left(\lambda_{1}-\lambda_{2}\right)^{2}+4 \lambda_{5}^{2}}\right)$

The second submatrix $\mathcal{M}_{3}$ corresponds to scattering with one of the following initial and final states: $\left(\phi_{1} \chi_{1}, \phi_{2} \chi_{2}\right)$. One finds that $\mathcal{M}_{3}$ is given by

$\mathcal{M}_{3}=\left(\begin{array}{ll}\lambda_{1} & \lambda_{5} \\ \lambda_{5} & \lambda_{2}\end{array}\right)$

the 3 eigenvalues read as follows:

$c_{ \pm}=f_{ \pm}$

The fourth submatrix $\mathcal{M}_{4}$ corresponds to scattering with initial and final states being one of the following 12 sates : $\left(\phi_{1} \phi_{1}^{+}, \chi_{1} \phi_{1}^{+}, \phi_{2} \phi_{1}^{+}, \chi_{2} \phi_{1}^{+}, \phi_{s} \phi_{1}^{+}, \phi_{1} \phi_{2}^{+}, \chi_{1} \phi_{2}^{+}, \phi_{2} \phi_{2}^{+}\right.$, $\left.\chi_{2} \phi_{2}^{+}, \phi_{s} \phi_{2}^{+}\right)$. It reads,

$\mathcal{M}_{4}$

$=\left(\begin{array}{cccccccccc}\lambda_{1} & 0 & 0 & 0 & 0 & 0 & 0 & \frac{\lambda_{45}^{+}}{2} & -i \frac{\lambda_{45}^{-}}{2} & 0 \\ 0 & \lambda_{1} & 0 & 0 & 0 & 0 & 0 & i \frac{\lambda_{45}^{-}}{2} & \frac{\lambda_{45}^{+}}{2} & 0 \\ 0 & 0 & \lambda_{3} & 0 & 0 & \frac{\lambda_{45}^{+}}{2} & i \frac{\lambda_{45}^{-}}{2} & 0 & 0 & 0 \\ 0 & 0 & 0 & \lambda_{3} & 0 & -i \frac{\lambda_{45}^{-}}{2} & \frac{\lambda_{45}^{+}}{2} & 0 & 0 & 0 \\ 0 & 0 & 0 & 0 & \frac{\lambda_{7}}{2} & 0 & 0 & 0 & 0 & 0 \\ 0 & 0 & \frac{\lambda_{45}^{+}}{2} & i \frac{\lambda_{45}^{-}}{2} & 0 & \lambda_{3} & 0 & 0 & 0 & 0 \\ 0 & 0 & -i \frac{\lambda_{45}^{-}}{2} & \frac{\lambda_{45}^{+}}{2} & 0 & 0 & \lambda_{3} & 0 & 0 & 0 \\ \frac{\lambda_{45}^{+}}{2} & -i \frac{\lambda_{45}^{-}}{2} & 0 & 0 & 0 & 0 & 0 & \lambda_{2} & 0 & 0 \\ i \frac{\lambda_{45}^{-}}{2} & \frac{\lambda_{45}^{+}}{2} & 0 & 0 & 0 & 0 & 0 & 0 & \lambda_{2} & 0 \\ 0 & 0 & 0 & 0 & 0 & 0 & 0 & 0 & 0 & \frac{\lambda_{8}}{2}\end{array}\right)$

The corresponding eigenvalues are:

$d_{1}=a_{1}, \quad d_{2}=a_{2}, \quad d_{3}=a_{3}, \quad d_{4}=a_{4}$ $d_{5}=a_{5}, \quad d_{ \pm}=b_{ \pm}, \quad g_{ \pm}=f_{ \pm}$

$d_{6}=\lambda_{3}-\lambda_{4}$

The fifth submatrix $\mathcal{M}_{5}$ corresponds to scattering with initial and final states being one of the following 3 sates: $\left(\frac{\phi_{1}^{+} \phi_{1}^{+}}{\sqrt{2}}, \frac{\phi_{2}^{+} \phi_{2}^{+}}{\sqrt{2}}, \phi_{1}^{+} \phi_{2}^{+}\right)$. It reads,

$\mathcal{M}_{5}=\left(\begin{array}{ccc}\lambda_{1} & \lambda_{5} & 0 \\ \lambda_{5} & \lambda_{2} & 0 \\ 0 & 0 & \lambda_{34}^{+}\end{array}\right)$

and possesses the following 3 distinct eigenvalues:

$e_{1}=a_{1}$

$e_{ \pm}=f_{ \pm}$

\section{Appendix C: Oblique parameters}

In order to study the contribution of the oblique parameters in the framework of N2HDM, we have used the general expressions presented in $[22,43,44]$ for the $S U(2) \times U(1)$ electroweak model with an arbitrary number of scalar $S U(2)$ doublets, with hypercharge $\pm \frac{1}{2}$, and an arbitrary number of scalar singlets.

In this study, we have four real fields that are related to the physical fields $h_{1,2,3}$ and $A$ through,

$$
\left(\begin{array}{c}
\varphi_{a}+i G \\
\varphi_{b}+i A \\
\varphi_{c}
\end{array}\right)=\left(\begin{array}{lllll}
i & R_{11} & R_{12} & R_{13} & 0 \\
0 & R_{21} & R_{22} & R_{23} & i \\
0 & R 31 & R_{32} & R_{23} & 0
\end{array}\right)\left(\begin{array}{c}
G \\
h_{1} \\
h_{2} \\
h_{3} \\
A
\end{array}\right)
$$

where $R_{i j}$ are defined in terms of the mixing angle $\beta$ and the elements of the rotation matrix given by eq, $\mathcal{R}_{i j}$, as follows,

$$
\begin{aligned}
& R_{11}=\mathcal{R}_{11} c_{\beta}+s_{\beta} \mathcal{R}_{12}, \quad R_{21}=\mathcal{R}_{12} c_{\beta}-s_{\beta} \mathcal{R}_{11}, \\
& R_{13}=\mathcal{R}_{13} \\
& R_{12}=\mathcal{R}_{21} c_{\beta}+\mathcal{R}_{22} s_{\beta}, \quad R_{22}=\mathcal{R}_{22} c_{\beta}-s_{\beta} \mathcal{R}_{21}, \\
& R_{32}=\mathcal{R}_{23} \\
& R_{13}=c_{\beta} \mathcal{R}_{31}+s_{\beta} \mathcal{R}_{32}, \quad R_{23}=\mathcal{R}_{32} c_{\beta}-s_{\beta} \mathcal{R}_{31}, \\
& R_{33}=\mathcal{R}_{33}
\end{aligned}
$$

We recall that the charged sector is the same as 2HDM, It contains only a pair of charged scalars $H^{ \pm}$. As a result the charged field is related to the physical charged scalar field through the unit matrix.

$$
\text { with } \tilde{\mu}^{2}=\frac{\mu^{2}}{s_{\beta} c_{\beta}} \text {. }
$$

Therefore, the oblique parameters $\mathrm{S}, \mathrm{T}$ and $\mathrm{U}$ in the N2HDM are given by:

$$
S=\frac{1}{24 \pi}\left[\left(2 s_{W}^{2}-1\right)^{2} G\left(m_{H^{+}}^{2}, m_{H^{+}}^{2}, m_{Z}^{2}\right)\right.
$$




$$
\begin{aligned}
+ & R_{21}^{2} G\left(m_{H_{1}}^{2}, m_{A}^{2}, m_{Z}^{2}\right) \\
& +R_{22}^{2} G\left(m_{H_{2}}^{2}, m_{A}^{2}, m_{Z}^{2}\right)+R_{23}^{2} G\left(m_{H_{3}}^{2}, m_{A}^{2}, m_{Z}^{2}\right) \\
& +\left(R_{11}^{2}+R_{21}^{2}\right) \ln \left(m_{H_{1}}^{2}\right) \\
& +\left(R_{12}^{2}+R_{22}^{2}\right) \ln \left(m_{H_{2}}^{2}\right)+\left(R_{13}^{2}+R_{23}^{2}\right) \ln \left(m_{H_{3}}^{3}\right) \\
& +\ln \left(m_{A}^{2}\right)-2 \ln \left(m_{H^{+}}^{2}\right)-\ln \left(m_{h_{r e f}}^{2}\right) \\
& +R_{11}^{2} \hat{G}\left(m_{H_{1}^{2}}^{2}, m_{Z}^{2}\right)+R_{12}^{2} \hat{G}\left(m_{H_{2}}^{2}, m_{Z}^{2}\right) \\
& \left.+R_{13}^{2} \hat{G}\left(m_{H_{3}}^{2}, m_{Z}^{2}\right)-\hat{G}\left(m_{h_{r e f}}^{2}, m_{Z}^{2}\right)\right] \\
T & \frac{1}{16 \pi^{2} m_{W}^{2} s_{W}^{2}}\left(R_{21}^{2} F\left(m_{H^{+}}^{2}, m_{H_{1}}^{2}\right)+R_{22}^{2} F\left(m_{H^{+}}^{2}, m_{H_{2}}^{2}\right)\right. \\
& +R_{23}^{2} F\left(m_{H^{+}}^{2}, m_{H_{3}}^{2}\right) \\
& +F\left(m_{H^{+}}^{2}, m_{A}^{2}\right)-R_{21}^{2} F\left(m_{H_{1}}^{2}, m_{A}^{2}\right)-R_{22}^{2} F\left(m_{H_{2}}^{2}, m_{A}^{2}\right) \\
& -R_{23}^{2} F\left(m_{H_{3}}^{2}, m_{A}^{2}\right) \\
& +3\left(R_{11}^{2}\left(F\left(m_{Z}^{2}, m_{H_{1}}^{2}\right)-F\left(m_{W}^{2}, m_{H_{1}}^{2}\right)\right)\right. \\
& +R_{12}^{2}\left(F\left(m_{Z}^{2}, m_{H_{2}}^{2}\right)-F\left(m_{W}^{2}, m_{H_{2}}^{2}\right)\right) \\
& \left.+R_{13}^{2}\left(F\left(m_{Z}^{2}, m_{H_{3}}^{2}\right)-F\left(m_{W}^{2}, m_{H_{3}}^{2}\right)\right)\right) \\
& \left.-3\left(F\left(m_{Z}^{2}, m_{h_{r e f}}^{2}\right)-F\left(m_{W}^{2}, m_{h_{r e f}}^{2}\right)\right)\right)
\end{aligned}
$$

and

$$
\begin{aligned}
U= & \frac{1}{24 \pi}\left[R_{21}^{2} G\left(m_{H^{+}}^{2}, m_{H_{1}}^{2}, m_{W}^{2}\right)\right. \\
& +R_{22}^{2} G\left(m_{H^{+}}^{2}, m_{H_{2}}^{2}, m_{W}^{2}\right) \\
& +R_{23}^{2} G\left(m_{H^{+}}^{2}, m_{H_{3}}^{2}, m_{W}^{2}\right)+G\left(m_{H^{+}}^{2}, m_{A}^{2}, m_{W}^{2}\right) \\
& -\left(2 s_{W}^{2}-1\right)^{2} G\left(m_{H^{+}}^{2}, m_{H^{+}}^{2}, m_{Z}^{2}\right) \\
& -\left(R_{21}^{2} G\left(m_{H_{1}}^{2}, m_{A}^{2}, m_{Z}^{2}\right)-R_{22}^{2} G\left(m_{H_{2}}^{2}, m_{A}^{2}, m_{Z}^{2}\right)\right. \\
& -R_{23}^{2} G\left(m_{H_{3}}^{2}, m_{A}^{2}, m_{Z}^{2}\right) \\
& +R_{11}^{2}\left(\hat{G}\left(m_{H_{1}}^{2}, m_{W}^{2}\right)-\hat{G}\left(m_{H_{1}}^{2}, m_{Z}^{2}\right)\right) \\
& +R_{12}^{2}\left(\hat{G}\left(m_{H_{2}}^{2}, m_{W}^{2}\right)\right. \\
& \left.-\hat{G}\left(m_{H_{2}}^{2}, m_{Z}^{2}\right)\right)+R_{13}^{2}\left(\hat{G}\left(m_{H_{3}}^{2}, m_{W}^{2}\right)-\hat{G}\left(m_{H_{3}}^{2}, m_{Z}^{2}\right)\right) \\
& \left.-G\left(m_{h_{r e f}}^{2}, m_{W}^{2}\right)+G\left(m_{h_{r e f}}^{2}, m_{Z}^{2}\right)\right]
\end{aligned}
$$

where $m_{h_{r e f}}$ is the reference mass of the neutral SM Higgs.
The explicit forms of these functions, $F(x, y), G(I, J, Q)$ and $\hat{G}(I, Q)$ are given by (C5), (C6) and (C7).

$$
\begin{aligned}
F(x, y) \equiv & \left\{\begin{array}{l}
\frac{x+y}{2}-\frac{x y}{x-y} \ln \frac{x}{y} \Leftarrow x \neq y, \\
0 \\
G
\end{array}\right. \\
& (I, J, Q)=-\frac{16}{3}+\frac{5(I+J)}{Q}-\frac{2(I-J)^{2}}{Q^{2}} \\
& +\frac{3}{Q}\left[\frac{I^{2}+J^{2}}{I-J}-\frac{I^{2}-J^{2}}{Q}+\frac{(I-J)^{3}}{3 Q^{2}}\right] \ln \frac{I}{J} \\
& +\frac{r}{Q^{3}} f(t, r) .
\end{aligned}
$$

If $I=J, G(I, J, Q)$ is:

$G(I, J, Q)=-\frac{16}{3}+\frac{16}{Q} I+\frac{r}{Q^{3}} f(t, r)$

and:

$$
f(t, r) \equiv\left\{\begin{aligned}
\sqrt{r} \ln \left|\frac{t-\sqrt{r}}{t+\sqrt{r}}\right| & \Leftarrow r>0, \\
0 & \Leftarrow r=0, \\
2 \sqrt{-r} \arctan \frac{\sqrt{-r}}{t} & \Leftarrow r<0 .
\end{aligned}\right.
$$

with:

$$
\begin{aligned}
t \equiv & I+J-Q \text { and } r \equiv Q^{2}-2 Q(I+J)+(I-J)^{2} \\
& \hat{G}(I, Q)=-\frac{79}{3}+9 \frac{I}{Q}-2 \frac{I^{2}}{Q^{2}} \\
& +\left(-10+18 \frac{I}{Q}-6 \frac{I^{2}}{Q^{2}}+\frac{I^{3}}{Q^{3}}-9 \frac{I+Q}{I-Q}\right) \ln \frac{I}{Q} \\
& +\left(12-4 \frac{I}{Q}+\frac{I^{2}}{Q^{2}}\right) \frac{f\left(I, I^{2}-4 I Q\right)}{Q} .
\end{aligned}
$$

\section{Appendix D: Scalar couplings}

We list hereafter the triple scalar couplings needed for our study (Table 1). 
Table 1 Trilinear Higgs boson self-interactions $(i \lambda 3 H)$ in the Feynman gauge within the N2HDM

\begin{tabular}{|c|c|}
\hline Vertex & Coupling \\
\hline$H_{1} H^{+} H^{-}$ & $-i \frac{1}{v}\left(-\tilde{\mu}^{2}\left(\frac{\mathcal{R}_{11}}{c_{\beta}}+\frac{\mathcal{R}_{12}}{s_{\beta}}\right)+m_{H_{1}}^{2}\left(\frac{\mathcal{R}_{11} s_{\beta}^{2}}{c_{\beta}}+\frac{\mathcal{R}_{12} c_{\beta}^{2}}{s_{\beta}}\right)+2 m_{H^{ \pm}}^{2}\left(\mathcal{R}_{11} c_{\beta}+\mathcal{R}_{12} s_{\beta}\right)\right)$ \\
\hline$H_{2} H^{+} H^{-}$ & $-i \frac{1}{v}\left(-\tilde{\mu}^{2}\left(\frac{\mathcal{R}_{21}}{c_{\beta}}+\frac{\mathcal{R}_{22}}{s_{\beta}}\right)+m_{H_{2}}^{2}\left(\frac{\mathcal{R}_{21} s_{\beta}^{2}}{c_{\beta}}+\frac{\mathcal{R}_{22} c_{\beta}^{2}}{s_{\beta}}\right)+2 m_{H^{ \pm}}^{2}\left(\mathcal{R}_{21} c_{\beta}+\mathcal{R}_{22} s_{\beta}\right)\right)$ \\
\hline$H_{3} H^{+} H^{-}$ & $-i \frac{1}{v}\left(-\tilde{\mu}^{2}\left(\frac{\mathcal{R}_{31}}{c_{\beta}}+\frac{\mathcal{R}_{32}}{s_{\beta}}\right)+m_{H_{3}}^{2}\left(\frac{\mathcal{R}_{31} s_{\beta}^{2}}{c_{\beta}}+\frac{\mathcal{R}_{32} c_{\beta}^{2}}{s_{\beta}}\right)+2 m_{H^{ \pm}}^{2}\left(\mathcal{R}_{31} c_{\beta}+\mathcal{R}_{32} s_{\beta}\right)\right)$ \\
\hline$H_{1} H_{1} H_{1}$ & $-i \frac{3}{v}\left(-\tilde{\mu}^{2}\left(\mathcal{R}_{12}^{2} c_{\beta}\left(\mathcal{R}_{12} \frac{c_{\beta}}{s_{\beta}}-\mathcal{R}_{11}\right)+\mathcal{R}_{11}^{2} s_{\beta}\left(\mathcal{R}_{11} \frac{s_{\beta}}{c_{\beta}}-\mathcal{R}_{12}\right)\right)+\frac{m_{H_{1}}^{2}}{v_{S}}\left(\mathcal{R}_{13}^{3} v+\mathcal{R}_{12}^{3} \frac{v_{S}}{s_{\beta}}+\mathcal{R}_{11}^{3} \frac{v_{S}}{c_{\beta}}\right)\right)$ \\
\hline $\mathrm{H}_{2} \mathrm{H}_{2} \mathrm{H}_{2}$ & $-i \frac{3}{v}\left(-\tilde{\mu}^{2}\left(\mathcal{R}_{22}^{2} c_{\beta}\left(\mathcal{R}_{22} \frac{c_{\beta}}{s_{\beta}}-\mathcal{R}_{21}\right)+\mathcal{R}_{21}^{2} s_{\beta}\left(\mathcal{R}_{21} \frac{s_{\beta}}{c_{\beta}}-\mathcal{R}_{22}\right)\right)+\frac{m_{H_{2}}^{2}}{v_{S}}\left(\mathcal{R}_{23}^{3} v+\mathcal{R}_{22}^{3} \frac{v_{S}}{s_{\beta}}+\mathcal{R}_{21}^{3} \frac{v_{S}}{c_{\beta}}\right)\right)$ \\
\hline $\mathrm{H}_{3} \mathrm{H}_{3} \mathrm{H}_{3}$ & $-i \frac{3}{v}\left(-\tilde{\mu}^{2}\left(\mathcal{R}_{32}^{2} c_{\beta}\left(\mathcal{R}_{32} \frac{c_{\beta}}{s_{\beta}}-\mathcal{R}_{31}\right)+\mathcal{R}_{31}^{2} s_{\beta}\left(\mathcal{R}_{31} \frac{s_{\beta}}{c_{\beta}}-\mathcal{R}_{32}\right)\right)+\frac{m_{H_{3}}^{2}}{v_{S}}\left(\mathcal{R}_{33}^{3} v+\mathcal{R}_{32}^{3} \frac{v_{S}}{s_{\beta}}+\mathcal{R}_{31}^{3} \frac{v_{S}}{c_{\beta}}\right)\right)$ \\
\hline$H_{2} H_{1} H_{1}$ & $\begin{array}{l}-i \frac{1}{v}\left(-\frac{1}{2} \tilde{\mu}^{2}\left(\frac{\mathcal{R}_{12}}{s_{\beta}}-\frac{\mathcal{R}_{11}}{c_{\beta}}\right)\left(6 \mathcal{R}_{12} \mathcal{R}_{22}+6 \mathcal{R}_{13} \mathcal{R}_{23} s_{\beta}^{2}+2 \mathcal{R}_{33} s_{\beta} c_{\beta}\right)+\frac{2 m_{H_{1}}^{2}+m_{H_{2}}^{2}}{v_{S}}\right. \\
\left.\left(\mathcal{R}_{13}^{2} \mathcal{R}_{23} v+\mathcal{R}_{12}^{2} \mathcal{R}_{22} \frac{v_{S}}{s_{\beta}}+\mathcal{R}_{11}^{2} \mathcal{R}_{21} \frac{v_{S}}{c_{\beta}}\right)\right)\end{array}$ \\
\hline$H_{3} H_{1} H_{1}$ & $\begin{array}{l}-i \frac{1}{v}\left(-\frac{1}{2} \tilde{\mu}^{2}\left(\frac{\mathcal{R}_{12}}{s_{\beta}}-\frac{\mathcal{R}_{11}}{c_{\beta}}\right)\left(6 \mathcal{R}_{12} \mathcal{R}_{32}+6 \mathcal{R}_{13} \mathcal{R}_{33} s_{\beta}^{2}-2 \mathcal{R}_{23} s_{\beta} c_{\beta}\right)+\frac{2 m_{H_{1}}^{2}+m_{H_{3}}^{2}}{v_{S}}\right. \\
\left.\left(\mathcal{R}_{13}^{2} \mathcal{R}_{33} v+\mathcal{R}_{12}^{2} \mathcal{R}_{32} \frac{v_{S}}{s_{\beta}}+\mathcal{R}_{11}^{2} \mathcal{R}_{31} \frac{v_{S}}{c_{\beta}}\right)\right)\end{array}$ \\
\hline$H_{3} H_{2} H_{2}$ & $\begin{array}{l}-i \frac{1}{v}\left(-\frac{1}{2} \tilde{\mu}^{2}\left(\frac{\mathcal{R}_{22}}{s_{\beta}}-\frac{\mathcal{R}_{21}}{c_{\beta}}\right)\left(6 \mathcal{R}_{22} \mathcal{R}_{32}+6 \mathcal{R}_{23} \mathcal{R}_{33} s_{\beta}^{2}+2 \mathcal{R}_{13} s_{\beta} c_{\beta}\right)+\frac{2 m_{H_{2}}^{2}+m_{H_{3}}^{2}}{v_{S}}\right. \\
\left.\left(\mathcal{R}_{23}^{2} \mathcal{R}_{33} v+\mathcal{R}_{22}^{2} \mathcal{R}_{32} \frac{v_{S}}{s_{\beta}}+\mathcal{R}_{21}^{2} \mathcal{R}_{31} \frac{v_{S}}{c_{\beta}}\right)\right)\end{array}$ \\
\hline
\end{tabular}

\section{References}

1. G. Aad et al., [ATLAS Collaboration], Observation of a new particle in the search for the Standard Model Higgs boson with the ATLAS detector at the LHC. Phys. Lett. B 716, 1 (2012). arXiv:1207.7214 [hep-ex]

2. S. Chatrchyan et al., [CMS Collaboration], Observation of a new boson at a mass of $125 \mathrm{GeV}$ with the CMS experiment at the LHC. Phys. Lett. B 716, 30 (2012). arXiv:1207.7235 [hep-ex]

3. M. Aaboud et al., [ATLAS Collaboration], Measurement of the Higgs boson mass in the $H \rightarrow Z Z^{*} \rightarrow 4 \ell$ and $H \rightarrow \gamma \gamma$ channels with $\sqrt{s}=13 \mathrm{TeV} p p$ collisions using the ATLAS detector. Phys. Lett. B 784, 345 (2018). arXiv: 1806.00242 [hep-ex]

4. A.M. Sirunyan et al., [CMS Collaboration], Measurements of properties of the Higgs boson decaying into the four-lepton final state in pp collisions at $\sqrt{s}=13 \mathrm{TeV}$. JHEP 1711, 047 (2017). arXiv:1706.09936 [hep-ex]

5. A. M. Sirunyan et al. [CMS Collaboration], Eur. Phys. J. C 79 (2019) no. 5, 421. https://doi.org/10.1140/epjc/ s10052-019-6909-y, arXiv:1809.10733 [hep-ex]

6. The ATLAS collaboration [ATLAS Collaboration], Combined measurements of Higgs boson production and decay using up to $80 \mathrm{fb}^{-1}$ of proton-proton collision data at $\sqrt{s}=13 \mathrm{TeV}$ collected with the ATLAS experiment, ATLAS-CONF-2018-031 (2019)

7. M. Aaboud et al., [ATLAS Collaboration], Constraints on off-shell Higgs boson production and the Higgs boson total width in $Z Z \rightarrow$ $4 \ell$ and $Z Z \rightarrow 2 \ell 2 v$ final states with the ATLAS detector. Phys. Lett. B 786, 223 (2018). arXiv:1808.01191 [hep-ex]

8. A. M. Sirunyan et al. [CMS Collaboration], Observation of tìtH production. Phys. Rev. Lett. 120, no. 23, 231801 (2018). arXiv:1804.02610 [hep-ex]
9. M. Aaboud et al., [ATLAS Collaboration], Observation of Higgs boson production in association with a top quark pair at the LHC with the ATLAS detector. Phys. Lett. B 784, 173 (2018). arXiv:1806.00425 [hep-ex]

10. A. M. Sirunyan et al. [CMS Collaboration], Observation of Higgs boson decay to bottom quarks," Phys. Rev. Lett. 121, no. 12, 121801 (2018) arXiv:1808.08242 [hep-ex]. M. Aaboud et al. [ATLAS Collaboration], "Observation of $H \rightarrow b \bar{b}$ decays and $V H$ production with the ATLAS detector," Phys. Lett. B 786 (2018) 59. arXiv: 1808.08238 [hep-ex]

11. A. Adhikary, S. Banerjee, R. Kumar Barman, B. Bhattacherjee, JHEP 1909 (2019) 068. https://doi.org/10.1007/ JHEP09(2019)068, arXiv:1812.05640 [hep-ph]

12. X. Cid Vidal et al. [Working Group 3], Beyond the Standard Model Physics at the HL-LHC and HE-LHC. arXiv:1812.07831 [hep-ph]

13. A. J. Costa, A. L. Carvalho, R. Gonalo, P. Muio, A. Onofre, Study of $t \bar{t} H$ production with $H \rightarrow b \bar{b}$ at the HL-LHC. arXiv:1812.10700 [hep-ph]

14. M. Cepeda et al. [HL/HE WG2 group], arXiv:1902.00134 [hep-ph]

15. S. Dawson et al., arXiv:1310.8361 [hep-ex]

16. D. Zeppenfeld, R. Kinnunen, A. Nikitenko, E. Richter-Was, Measuring Higgs boson couplings at the CERN LHC. Phys. Rev. D 62, 013009 (2000). arXiv:hep-ph/0002036

17. F. Gianotti, M. Pepe-Altarelli, Precision physics at the LHC. Nucl. Phys. Proc. Suppl. 89, 177 (2000). arXiv:hep-ex/0006016

18. C. Englert, A. Freitas, M.M. Mühlleitner, T. Plehn, M. Rauch, M. Spira, K. Walz, Precision measurements of Higgs Couplings: implications for new physics scales. J. Phys. G 41, 113001 (2014). arXiv:1403.7191 [hep-ph]

19. G. Moortgat-Pick et al., Physics at the e+ e- Linear Collider. Eur. Phys. J. C 75(8), 371 (2015). arXiv:1504.01726 [hep-ph] 
20. T. Robens, T. Stefaniak, Status of the Higgs Singlet extension of the standard model after LHC Run 1. Eur. Phys. J. C 75, 104 (2015). arXiv:1501.02234 [hep-ph]

21. T.D. Lee, A Theory of Spontaneous T Violation. Phys. Rev. D 8, $1226(1973)$

22. J.F. Gunion, H.E. Haber, The CP conserving two Higgs doublet model: the approach to the decoupling limit. Phys. Rev. D 67, 075019 (2003). arXiv:hep-ph/0207010

23. G.C. Branco, P.M. Ferreira, L. Lavoura, M.N. Rebelo, M. Sher, J.P. Silva, Theory and phenomenology of two-Higgs-doublet models. Phys. Rept. 516, 1 (2012). https://doi.org/10.1016/j.physrep.2012. 02.002

24. N.G. Deshpande, E. Ma, Pattern of symmetry breaking with two Higgs doublets. Phys. Rev. D 18, 2574 (1978)

25. E. Ma, Verifiable radiative seesaw mechanism of neutrino mass and dark matter. Phys. Rev. D 73, 077301 (2006). arXiv:hep-ph/0601225

26. R. Barbieri, L.J. Hall, V.S. Rychkov, Improved naturalness with a heavy Higgs: an alternative road to LHC physics. Phys. Rev. D 74, 015007 (2006). arXiv:hep-ph/0603188

27. Q.H. Cao, E. Ma, G. Rajasekaran, Observing the dark scalar doublet and its impact on the standard-model Higgs Boson at colliders. Phys. Rev. D 76, 095011 (2007). arXiv:0708.2939 [hep-ph]

28. A. Drozd, B. Grzadkowski, J.F. Gunion, Y. Jiang, Extending twoHiggs-doublet models by a singlet scalar field- the Case for Dark Matter. JHEP 1411, 105 (2014). arXiv:1408.2106 [hep-ph]

29. B. Grzadkowski, P. Osland, Tempered Two-Higgs-Doublet model. Phys. Rev. D 82, 125026 (2010). arXiv:0910.4068 [hep-ph]

30. C.Y. Chen, M. Freid, M. Sher, Next-to-minimal two Higgs doublet model. Phys. Rev. D 89(7), 075009 (2014). arXiv:1312.3949 [hep$\mathrm{ph}]$

31. M. Mühlleitner, M.O.P. Sampaio, R. Santos, J. Wittbrodt, The N2HDM under theoretical and experimental scrutiny. JHEP 1703, 094 (2017). arXiv:1612.01309 [hep-ph]

32. G. Aad et al. [ATLAS Collaboration], Eur. Phys. J. C 76(4), 210 (2016). https://doi.org/10.1140/epjc/s10052-016-4034-8, arXiv:1509.05051 [hep-ex]

33. S.L. Glashow, S. Weinberg, Natural conservation laws for neutral currents. Phys. Rev. D 15, 1958 (1977). https://doi.org/10.1103/ PhysRevD.15.1958

34. G.C. Branco, P.M. Ferreira, L. Lavoura, M.N. Rebelo, M. Sher, J.P. Silva, Theory and phenomenology of two-Higgs-doublet models. Phys. Rept. 516, 1 (2012). arXiv:1106.0034 [hep-ph]

35. J.F. Gunion, H.E. Haber, J. Wudka, Sum rules for Higgs bosons. Phys. Rev. D 43, 904 (1991)

36. M.P. Bento, H.E. Haber, J.C. Romão, J.P. Silva, Multi-Higgs doublet models: physical parametrization, sum rules and unitarity bounds. JHEP 1711, 095 (2017). arXiv:1708.09408 [hep-ph]

37. M. P. Bento, H. E. Haber, J. C. Romão, J. P. Silva, JHEP 1810, 143 (2018). https://doi.org/10.1007/JHEP10(2018)143, arXiv:1808.07123 [hep-ph]

38. S. Kanemura, T. Kubota, E. Takasugi, Lee-Quigg-Thacker bounds for Higgs boson masses in a two doublet model. Phys. Lett. B 313, 155 (1993). arXiv:hep-ph/9303263

39. A.G. Akeroyd, A. Arhrib, E.M. Naimi, Note on tree level unitarity in the general two Higgs doublet model. Phys. Lett. B 490, 119 (2000). arXiv:hep-ph/0006035

40. A. Arhrib, Unitarity constraints on scalar parameters of thestandard and two Higgs doublets model (2019). arXiv:hep-ph/0012353

41. M.E. Peskin, T. Takeuchi, A New constraint on a strongly interacting Higgs sector. Phys. Rev. Lett. 65, 964 (1990)

42. M.E. Peskin, T. Takeuchi, Estimation of oblique electroweak corrections. Phys. Rev. D 46, 381 (1992)

43. W. Grimus, L. Lavoura, O.M. Ogreid, P. Osland, A Precision constraint on multi-Higgs-doublet models. J. Phys. G 35, 075001 (2008). arXiv:0711.4022 [hep-ph]
44. W. Grimus, L. Lavoura, O.M. Ogreid, P. Osland, The Oblique parameters in multi-Higgs-doublet models. Nucl. Phys. B 801, 81 (2008). arXiv:0802.4353 [hep-ph]

45. J. Haller, A. Hoecker, R. Kogler, K. Mnig, T. Peiffer and J. Stelzer, Update of the global electroweak fit and constraints on two-Higgs-doublet models. Eur. Phys. J. C 78(8), 675 (2018). arXiv:1803.01853 [hep-ph]

46. H.J. He, N. Polonsky, Sf Su, Extra families, Higgs spectrum and oblique corrections. Phys. Rev. D 64, 053004 (2001). arXiv:hep-ph/0102144

47. S. Kanemura, Y. Okada, H. Taniguchi, K. Tsumura, Indirect bounds on heavy scalar masses of the two-Higgs-doublet model in light of recent Higgs boson searches. Phys. Lett. B 704, 303 (2011). arXiv:1108.3297 [hep-ph]

48. Y. Amhis et al. [HFLAV Collaboration], Averages of $b$-hadron, $c$ hadron, and $\tau$-lepton properties as of summer 2016. Eur. Phys. J. C 77(12), 895 (2017). arXiv:1612.07233 [hep-ex]

49. M. Misiak, M. Steinhauser, Eur. Phys. J. C 77(3), 201 (2017). https://doi.org/10.1140/epjc/s10052-017-4776-y, arXiv:1702.04571 [hep-ph]

50. P. Bechtle, O. Brein, S. Heinemeyer, G. Weiglein, K.E. Williams, HiggsBounds: confronting arbitrary Higgs sectors with exclusion bounds from LEP and the Tevatron. Comput. Phys. Commun. 181, 138 (2010). arXiv:0811.4169 [hep-ph]

51. P. Bechtle, O. Brein, S. Heinemeyer, G. Weiglein, K.E. Williams, HiggsBounds 2.0.0: confronting neutral and charged Higgs sector predictions with exclusion bounds from LEP and the Tevatron. Comput. Phys. Commun. 182, 2605 (2011). arXiv:1102.1898 [hep$\mathrm{ph}]$

52. P. Bechtle, O. Brein, S. Heinemeyer, O. Stål, T. Stefaniak, G. Weiglein, K.E. Williams, HiggsBounds - 4: improvedTests of Extended Higgs Sectors against Exclusion Bounds from LEP, the Tevatron and the LHC. Eur. Phys. J. C 74(3), 2693 (2014). arXiv:1311.0055 [hep-ph]

53. P. Bechtle, S. Heinemeyer, O. Stål, T. Stefaniak, G. Weiglein, Higgs Signals: confronting arbitrary Higgs sectorswith measurements at the Tevatron and the LHC. Eur. Phys. J. C 74(2), 2711 (2014). arXiv:1305.1933 [hep-ph]

54. V. Khachatryan et al., [CMS Collaboration], Searches for invisible decays of the Higgs boson in pp collisions at $\sqrt{s}=7,8$, and 13 TeV. JHEP 1702, 135 (2017). arXiv:1610.09218 [hep-ex]

55. M. Aaboud et al., [ATLAS Collaboration], Search for an invisibly decaying Higgs boson or dark matter candidates produced in association with a $Z$ boson in $p p$ collisions at $\sqrt{s}=13 \mathrm{TeV}$ with the ATLAS detector. Phys. Lett. B 776, 318 (2018). arXiv:1708.09624 [hep-ex]

56. A.M. Sirunyan et al., [CMS Collaboration]. Phys. Lett. B 793, 520 (2019). https://doi.org/10.1016/j.physletb.2019.04.025, arXiv:1809.05937 [hep-ex]

57. A.M. Sirunyan et al., [CMS Collaboration], Phys. Lett. B 785, 462 (2018). https://doi.org/10.1016/j.physletb.2018.08.057, arXiv:1805.10191 [hep-ex]

58. M. Aaboud et al. [ATLAS Collaboration], Eur. Phys. J. C 76(11), 605 (2016). https://doi.org/10.1140/epjc/s10052-016-4418-9, arXiv:1606.08391 [hep-ex]

59. M. Mühlleitner, M.O.P. Sampaio, R. Santos, J. Wittbrodt, Phenomenological comparison of models with extended Higgs sectors. JHEP 1708, 132 (2017). arXiv:1703.07750 [hep-ph]

60. D. Azevedo, P. Ferreira, M.M. Mühlleitner, R. Santos, J. Wittbrodt, Phys. Rev. D 99(5), 055013 (2019). https://doi.org/10.1103/ PhysRevD.99.055013. arXiv:1808.00755 [hep-ph]

61. I. Engeln, M. Mühlleitner, J. Wittbrodt, N2HDECAY: Higgs Boson decays in the different phases of the N2HDM. Comput. Phys. Commun. 234, 256 (2019). arXiv:1805.00966 [hep-ph]

62. S. von Buddenbrock, A.S. Cornell, E.D.R. Iarilala, M. Kumar, B. Mellado, X. Ruan, E.M. Shrif, J. Phys. G 46(11), 115001 (2019). 
https://doi.org/10.1088/1361-6471/ab3cf6. arXiv:1809.06344 [hep-ph]

63. A. Djouadi, J. Kalinowski, M. Spira, Comput. Phys. Commun. 108, 56 (1998). https://doi.org/10.1016/S0010-4655(97)00123-9. arXiv:hep-ph/9704448

64. J. Bernon, J.F. Gunion, H.E. Haber, Y. Jiang, S. Kraml, Phys. Rev. D 93(3), 035027 (2016). arXiv:1511.03682 [hep-ph]

65. A. Arhrib, R. Benbrik, S. Moretti, A. Rouchad, Q.S. Yan, X. Zhang, JHEP 1807, 007 (2018). arXiv:1712.05332 [hep-ph]
66. R.V. Harlander, S. Liebler, H. Mantler, Comput. Phys. Commun. 184, 1605 (2013). arXiv:1212.3249 [hep-ph]

67. R.V. Harlander, S. Liebler, H. Mantler, Comput. Phys. Commun. 212, 239 (2017). arXiv:1605.03190 [hep-ph]

68. A. M. Sirunyan et al. [CMS Collaboration], Phys. Rev. Lett. 122(12), 121803 (2019). arXiv:1811.09689 [hep-ex] 